ARTICLE

\title{
Spatial control of avidity regulates initiation and progression of selective autophagy
}

\author{
David M. Hollenstein (1) 1,2,3,7, Mariya Licheva (10 3,4,7, Nicole Konradi ${ }^{3}$, David Schweida ${ }^{1}$, Hector Mancilla ${ }^{3}$,
} Muriel Mari (iD ${ }^{5}$, Fulvio Reggiori (iD ${ }^{5}$ \& Claudine Kraft (iD ${ }^{3,6 \times}$

Autophagosomes form at the endoplasmic reticulum in mammals, and between the vacuole and the endoplasmic reticulum in yeast. However, the roles of these sites and the mechanisms regulating autophagosome formation are incompletely understood. Vac8 is required for autophagy and recruits the Atg1 kinase complex to the vacuole. Here we show that Vac8 acts as a central hub to nucleate the phagophore assembly site at the vacuolar membrane during selective autophagy. Vac8 directly recruits the cargo complex via the Atg11 scaffold. In addition, Vac8 recruits the phosphatidylinositol 3-kinase complex independently of autophagy. Cargo-dependent clustering and Vac8-dependent sequestering of these early autophagy factors, along with local Atg1 activation, promote phagophore assembly site assembly at the vacuole. Importantly, ectopic Vac8 redirects autophagosome formation to the nuclear membrane, indicating that the vacuolar membrane is not specifically required. We propose that multiple avidity-driven interactions drive the initiation and progression of selective autophagy.

\footnotetext{
${ }^{1}$ Department of Biochemistry and Cell Biology, Max Perutz Labs, University of Vienna, Vienna BioCenter (VBC), Dr. Bohr-Gasse 9, 1030 Vienna, Austria.

${ }^{2}$ Vienna BioCenter PhD Program, Doctoral School of the University of Vienna and Medical University of Vienna, Vienna, Austria. ${ }^{3}$ Institute of Biochemistry and Molecular Biology, ZBMZ, Faculty of Medicine, University of Freiburg, 79104 Freiburg, Germany. ${ }^{4}$ Faculty of Biology, University of Freiburg, 79104 Freiburg, Germany. ${ }^{5}$ Department of Biomedical Sciences of Cells \& Systems, University of Groningen, University Medical Center Groningen, Groningen, The Netherlands. ${ }^{6}$ CIBSS - Centre for Integrative Biological Signalling Studies, University of Freiburg, 79104 Freiburg, Germany. ${ }^{7}$ These authors contributed

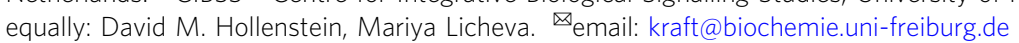


$\mathrm{M}$ acroautophagy, hereafter referred to as autophagy, is an intracellular degradation and recycling pathway that is highly conserved among eukaryotes. During autophagy, cellular material referred to as cargo is engulfed within a newly formed double membrane vesicle, the autophagosome. Once complete, the outer autophagosomal membrane fuses with the lytic compartment (the lysosome in mammals or the vacuole in yeast and plants) releasing the inner vesicle and its cargo for degradation and recycling.

Autophagy is initiated by the formation of a transient structure called the phagophore assembly site (PAS, also known as the preautophagosomal structure), which defines the cellular location of autophagosome formation ${ }^{1,2}$. The PAS forms differently depending on the type of autophagy: selective or nonselective. Whereas cytoplasmic material is nonspecifically sequestered by autophagosomes in response to starvation during nonselective or "bulk" autophagy, selective autophagy involves the specific recognition of a cargo by dedicated receptors ${ }^{3,4}$. PAS initiation in bulk autophagy does not require cargo, whereas PAS formation in selective autophagy is triggered by the assembly of the core autophagy machinery on cargo-receptor complexes.

The cytoplasm-to-vacuole targeting (Cvt) pathway is a biosynthetic selective autophagy pathway in S. cerevisiae that delivers aminopeptidase 1 (Ape1) oligomers to the vacuole. The cargo receptor autophagy-related 19 (Atg19) binds to both the Ape1 cargo and the scaffold protein Atg1 $1^{5}$. Subsequently, the Atg11bound cargo is recruited to the vacuole, where it colocalizes with the Atg14-containing PI3-kinase complex 1 (PI3KC1) into a distinct structure as part of the PAS 1,6 . Atg11 also recruits the Atg1-kinase complex to the $\mathrm{PAS}^{7-9}$. Similarly in mammals, FIP200-bound cargo and PI3KC3-C1 (class-3 PI3KC1) are both recruited to specific ER domains, where they colocalize during PAS and autophagosome formation. FIP200 is also required for recruitment of the ULK1 kinase complex ${ }^{10-12}$. Nevertheless, it remains unknown how Atg11/FIP200-bound cargo and the PI3K complex are recruited to membranes, the vacuole or ER respectively, and whether this specific localization fulfills a functional role during PAS assembly and autophagosome formation.

Vac8 is a vacuole-anchored protein known for its role in vacuole inheritance, homotypic vacuole fusion, and nucleus-vacuole junctions, but has also been found to be required for both selective and bulk autophagy function ${ }^{13-18}$. During bulk autophagy, Vac8 binds Atg13, a subunit of the Atg1 kinase complex ${ }^{16}$, which results in the recruitment of other Atg proteins to assemble the bulk PAS at the vacuole ${ }^{19-22}$. Atg13, however, is dispensable for the recruitment of Atg11-bound cargo complexes to the vacuole ${ }^{8}$ and thus dispensable for the initiation of selective PAS formation. It therefore remains unclear which role Vac8 plays during selective autophagy.

Detailed insight into the regulation of PAS assembly and the role of membranes has been obscured due to technical limitations. Classical in vitro systems provide the advantage of a chemically well-defined environment but may not sufficiently recapitulate essential cellular contexts, such as distinct protein localizations or membrane compositions ${ }^{23,24}$. On the other hand, in vivo approaches also have limitations, as for instance deletion of a PAS-assembly factor will compromise its earliest role in the assembly process, effectively masking parallel or later requirements. Therefore, the interplay and dependencies of PASassembly factors and the importance of their subcellular location have largely remained elusive.

To overcome this limitation, we developed a synthetic reconstitution approach in intact cells that allowed us to systematically dissect the regulatory principles of PAS assembly. Using this system, we discovered that the mechanisms underlying PAS formation at the vacuole are based on avidity, achieved by clustering and compartmentalization. We found that Vac8 serves as a central hub to confine and coordinate the cargo and multiple autophagy factors at the vacuole in parallel, and thus is required to initiate autophagosome formation. Strikingly, we were able to rescue selective autophagy-defective vac $8 \Delta$ yeast mutants by redirecting PAS assembly to the nuclear membrane with an ectopic Vac8.

\section{Results}

Vac8 anchors the selective PAS at the vacuole. Cargo receptors bind to Atg11, which serves as the scaffold to initiate selective autophagy. Atg11 anchors the cargo-receptor complex to the vacuole and recruits other autophagy factors, such as Atg1, to form the PAS ${ }^{5,25}$. How Atg11 is recruited to the vacuole remains unknown. To determine if other Atg proteins are required to anchor Atg11 at the vacuole, we tested seven Atg proteins from the main functional groups: Atg1 and Atg13 (Atg1/ULK1 kinase complex), Atg9 and Atg2 (Atg9 and Atg2-Atg18 system), Atg14 (PI3KC1), Atg12 (Atg12-Atg5-Atg16 conjugation system), and Atg8. We monitored the localization of the fluorescently labeled selective autophagy cargo BFP-Ape1, which was expressed in the respective deletion mutant strains. As expected, deletion of Atg11 abrogated BFP-Ape1 recruitment to the vacuole, detected by staining with FM4-64,25. In contrast, BFP-Ape1 was recruited to the vacuole in $\operatorname{atg} 1 \Delta, \operatorname{atg} 13 \Delta, \operatorname{atg} 9 \Delta, \operatorname{atg} 14 \Delta, \operatorname{atg} 12 \Delta, \operatorname{atg} 2 \Delta$, and atg $8 \Delta$ mutants, similar to wild-type cells (Fig. 1a, b and Supplementary Fig. 1a). Thus, canonical Atg proteins are not required to anchor Atg11-bound cargo complexes at the vacuole.

Cargo recognition by autophagy receptor proteins results in local clustering of Atg11 on the cargo complex. To test if clustering of Atg11 is sufficient for its vacuolar localization, we fused Atg11-GFP to a fragment of the reoviral nonstructural protein $\mu \mathrm{NS}$, which forms multimeric structures of heterogeneous size when expressed in yeast ${ }^{26}$ (Fig. $1 \mathrm{c}$ and Supplementary Fig. 1b). Both Atg11-GFP- $\mu$ NS and control GFP- $\mu$ NS accumulated mostly as a large single cytosolic punctum in $\operatorname{atg} 11 \Delta \operatorname{atg} 19 \Delta$

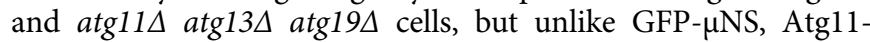
GFP- $\mu$ NS was always associated with the vacuole (Fig. 1d, e). Moreover, we observed a dramatic bending of the vacuolar membrane around Atg11-GFP- $\mu \mathrm{NS}$, suggesting that multiple interactions between Atg11-GFP- $\mu$ NS and a vacuolar binding partner deform the vacuolar membrane. Atg11-GFP- $\mu$ NS particles were not taken up into the vacuolar lumen but remained attached to the outer leaflet of the vacuolar membrane. Importantly, the Atg11-GFP- $\mu$ NS particles maintained characteristics of endogenous Atg11, such as binding to Atg1, Atg9 and Atg19, as well as its ability to self-interact (Supplementary Fig. 1c, d). Moreover, the interaction of Atg11-GFP- $\mu$ NS with the vacuole was independent of the cargo-receptor complex (Fig. 1d). Atg11GFP- $\mu$ NS therefore serves as an ideal tool to study vacuolar recruitment of Atg11 in vivo.

Given that Vac8 recruits Atg13 to the vacuole to anchor the bulk PAS, we hypothesized that Vac8 might also interact with Atg11 to facilitate selective PAS assembly. To test this hypothesis, we monitored localization of Vac8-mCherry in cells coexpressing Atg11-GFP- $\mu N S$, or Atg13-GFP- $\mu$ NS as a control. Clustered Atg13-GFP- $\mu$ NS localized to the vacuole and recruited Vac8mCherry, leading to its depletion from the rest of the vacuolar membrane (Fig. 1f). Intriguingly, Vac8-mCherry was also redistributed to the vacuolar contact site of Atg11-GFP- $\mu$ NS, suggesting that Atg11-GFP- $\mu$ NS specifically recruits Vac8 to this location (Fig. 1f). In contrast, the vacuolar V-type proton ATPase Vph1-mCherry was displaced from Atg11-GFP- $\mu$ NS contact sites (Fig. 1g). These findings suggest that Vac8 interacts with Atg11, recruiting it to the vacuole. 
a

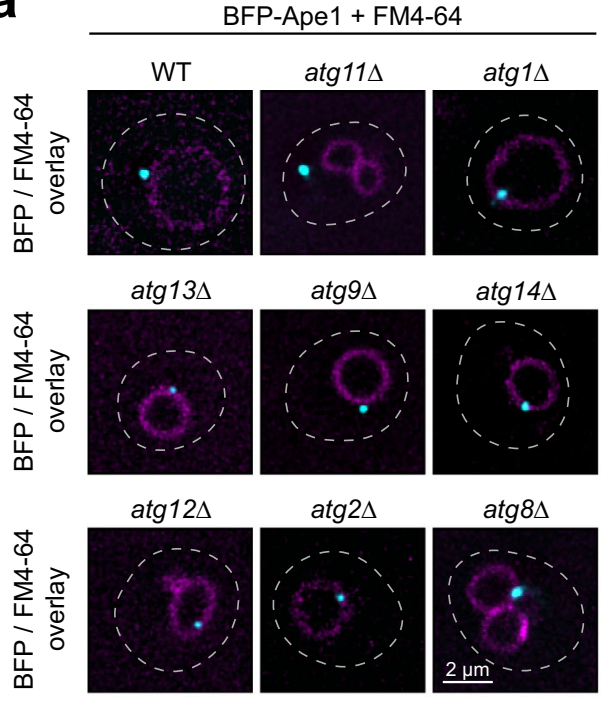

d



b

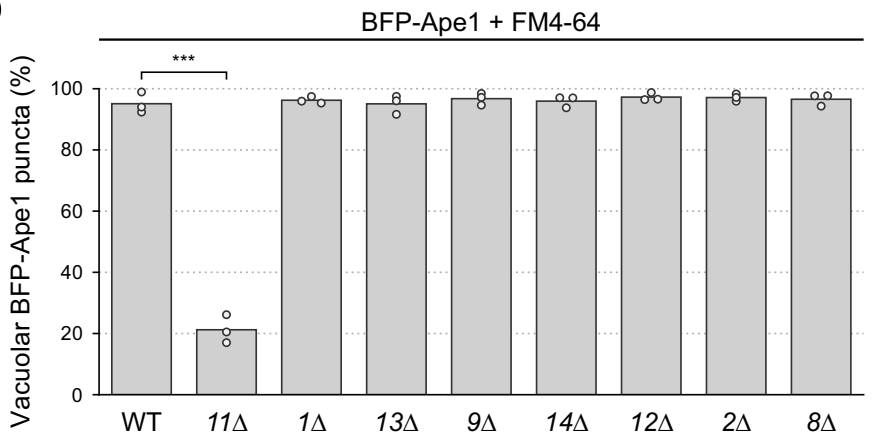

C

$$
\operatorname{Atg} 11-\mu N S
$$

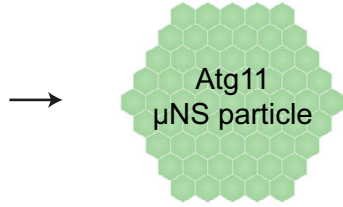

e

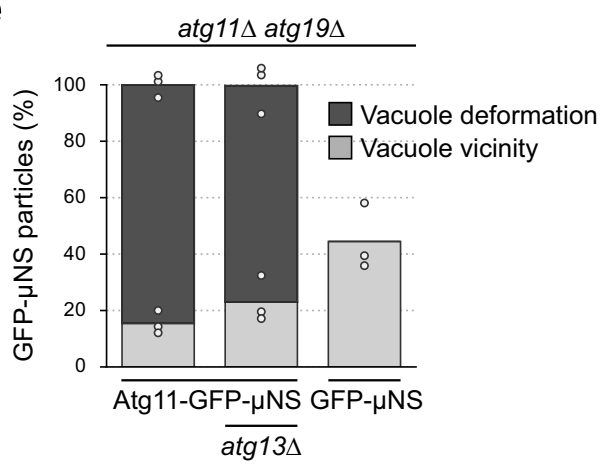

f

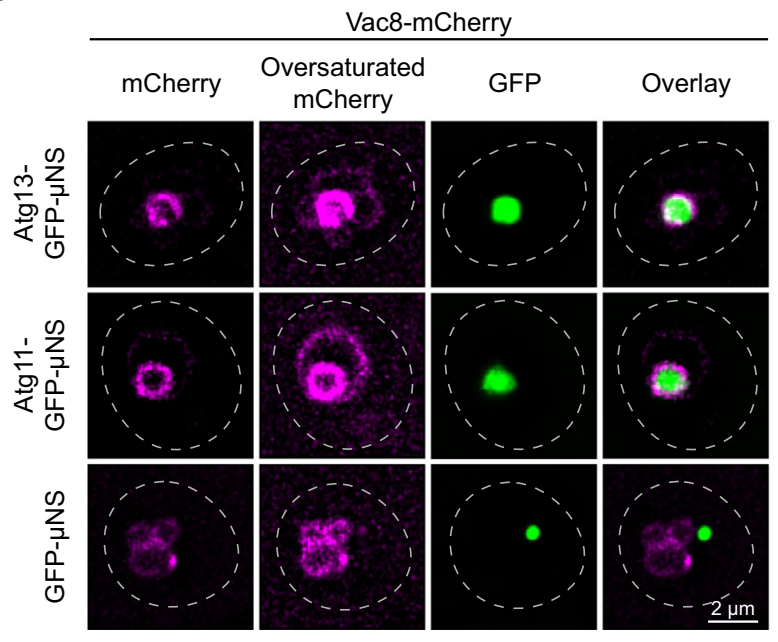

g

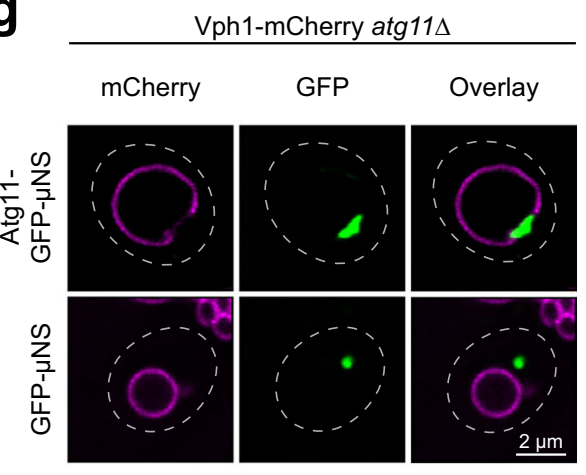

To test if Vac8 is required to anchor the Atg11-cargo complex at the vacuole, we monitored BFP-Apel localization in vac8D cells. In wild-type cells, most BFP-Ape1 puncta were localized in proximity to Vph1-mCherry-containing vacuoles. This localization was largely lost in atg $11 \Delta$ mutants, as expected. vac $8 \Delta$ cells showed vacuolar fragmentation, as previously reported ${ }^{27}$. Importantly, vac $8 \Delta$ cells also displayed a severe defect in vacuole recruitment of BFP-Apel puncta, however less pronounced than in atg11 $\Delta$ cells. Deletion of both, Atg11 and Vac8, did not result in a more severe vacuole-recruitment defect of BFP-Ape1 puncta than a single deletion of VAC8 (Fig. 2a, b and Supplementary Fig. 2a), indicating that the vacuole fragmentation phenotype of vac8s cells leads to an increase of false positives in the quantification of vacuole localized puncta, as previously 
Fig. 1 Vac8 clusters at the PAS-vacuole contact site. a and $\mathbf{b}$ Wild-type (WT) or indicated atg $\triangle$ strains containing TagBFP-Ape1 were grown to mid-log phase, and vacuoles were stained with FM4-64. a Representative microscopy images showing the overlay of BFP and FM4-64. Dashed lines indicate the contour of individual cells. See also Supplementary Fig. 1a. b The number of vacuolar BFP-Ape1 puncta was quantified in three independent experiments. For each strain and replicate at least 120 BFP puncta were analyzed. The values of each replicate (circle) and the mean (bars) were plotted. Statistical analysis using two-tailed unpaired $t$-tests. Significance is indicated with asterisks: ${ }^{\star \star \star} p<0.001,{ }^{\star \star} p<0.01,{ }^{\star} p<0.05$, n.s. (not significant) $p>0.05$. Exact numerical values are reported in the source data. c Schematic illustration of the Atg11- $\mu$ NS particle, formed from single monomeric subunits. Note that these particles vary in size and that their structure is unknown. $\mathbf{d}$ and $\mathbf{e}$ atg $11 \Delta$ atg19 $\Delta$ and $\operatorname{atg} 11 \Delta$ atg $13 \Delta$ atg $19 \Delta$ cells containing Atg11-GFP- $\mu$ NS or GFP- $\mu$ NS were grown to mid-log phase. Vacuoles were stained with FM4-64. $\mu$ NS $=$ amino acids 471-721 of the reoviral nonstructural protein $\mu$ NS. $\mathbf{d}$ Representative microscopy images are shown. Dashed lines indicate the contour of individual cells. e Vacuolar deformation or vacuolar vicinity of Atg11-GFP- $\mu$ NS and GFP$\mu N S$ particles was quantified in three independent experiments. For each strain and replicate at least $70 \mu N S$ particles were analyzed. The values of each replicate (circle) and the mean (bars) were plotted. See also Supplementary Fig. 1b. f Vac8-mCherry cells expressing Atg13-GFP- $\mu$ NS, Atg11-GFP- $\mu$ NS or GFP- $\mu$ NS were grown to mid-log phase. The intracellular localization of the $\mu$ NS fusion proteins and the vacuolar distribution of Vac 8 was monitored by fluorescence microscopy. Oversaturated images of the mCherry signal allow complete visualization of the vacuolar membrane. Representative fluorescence microscopy images of one out of three independent experiments are shown. Dashed lines indicate the contour of individual cells. $\mathbf{g}$ Vph1$4 \times m$ Cherry atg $11 \Delta$ cells containing Atg11-GFP- $\mu$ NS or GFP- $\mu$ NS were grown to mid-log phase. Representative fluorescence microscopy images of one out of three independent experiments are shown. Dashed lines indicate the contour of individual cells.

observed $^{21}$. Atg11-GFP- $\mu$ NS particles also failed to localize to the vacuolar membrane and induce vacuolar membrane bending in vac8 $\Delta$ cells (Supplementary Fig. 2b). To investigate the vacuolar contact of Atg11 at a higher resolution, we analyzed cells expressing Atg11-GFP- $\mu$ NS or GFP- $\mu$ NS by transmission electron microscopy (TEM). Clear contacts of Atg11-GFP- $\mu$ NS spreading along the vacuolar surface were observed in over $80 \%$ of cells expressing Atg11-GFP- $\mu$ NS, whereas such contacts were absent in cells expressing GFP- $\mu$ NS. These contacts were also absent in vac $8 \Delta$ cells expressing Atg11-GFP- $\mu$ NS (Fig. $2 c$, d). Overall, these data demonstrate that $\operatorname{Vac} 8$ is required to recruit Atg11 complexes to the vacuolar membrane.

The interaction of Atg11 with Vac8 is direct and requires clustering. To decipher which region of Atg11 is required for vacuolar anchoring, we generated a series of Atg11 truncation mutants. We fused these truncations to GFP-Atg19, as they lack the Atg19 binding domain (Fig. $2 \mathrm{e}^{8}$ ). We analyzed GFP localization in atg11 $\operatorname{atg} 19 \Delta$ cells and observed that all truncations formed distinct cytosolic puncta, indicating the efficient recruitment of the fusion proteins to Apel oligomers (Supplementary Fig. 3a). As expected, full-length Atg11-GFP-Atg19 puncta localized to the vacuole, marked with Sna3-mCherry, whereas GFPAtg19 puncta were mostly cytoplasmic without vacuolar contacts (Fig. 2f and Supplementary Fig. 3a). We found that the N-terminal 454 amino acids of Atg11 were necessary and sufficient for vacuolar localization. In addition, we fused Atg11 1-454 to GFP- $\mu$ NS and observed vacuolar invaginations and clustering of $\mathrm{Vac} 8$ at the vacuole contact site of the particles, similar to fulllength Atg11-GFP- $\mu$ NS (Supplementary Fig. 3b).

To investigate a potential physical association between Vac8 and Atg11, we performed co-immunoprecipitation experiments. We found that full-length Atg11 and Atg11 $1-454$ coimmunoprecipitated with Vac8-GFP (Fig. 2g), independently of Atg13 (Supplementary Fig. 3c). In contrast, the known Atg11 interactors Atg1, Atg9, Atg17, Atg19, and Atg29 only coimmunoprecipitated with protein A-tagged full-length Atg11 (PrA-Atg11 ${ }^{\mathrm{FL}}$ ), but not PrA-Atg11 $1^{1-454}$ (Supplementary Fig. 3d). To test if Vac8 and Atg11 interact directly, we performed GST pull-down experiments. Indeed, purified GST-Vac8 bound to His-Atg11 in insect cell lysate, suggesting that this interaction is direct (Fig. 3a). Similarly, Vac8 and Atg11 interacted in yeast-two hybrid analyses, and Atg11 1 -454 was sufficient for this interaction (Supplementary Fig. 4a). The Atg11-Vac8 interaction, however, was very weak compared with the known interaction of Atg11 with $\operatorname{Atg} 19^{3 \mathrm{D}}$ (Fig. 3 $\mathrm{b}^{28}$ ). Together, these findings demonstrate that the $\mathrm{N}$ terminus of Atg11 interacts directly with Vac8, however, with low affinity.

We hypothesized that if endogenous Vac8 anchors Atg11 at the vacuole, then ectopic localization of Vac8 should anchor Atg11 to another site in the cell. To this end, we employed a tethering system to generate a cytosolic Vac8 site. Briefly, we fused a cytosolic Vac8 truncation mutant to an $\mathrm{N}$-terminal peptide that confers Ape1 binding (ot-Vac8 $\triangle \mathrm{N}-\mathrm{BFP}$; ot = oligomer tether), and expressed this construct in atg19D cells ${ }^{5,29}$. (Fig. 3c). In this system, Ape1 oligomers are cytosolic and do not interact with other Atg proteins, but induce the clustering of ot-Vac $8 \Delta \mathrm{N}-\mathrm{BFP}$. Therefore, these oligomers can be repurposed to provide a protein-clustering platform that enables the visualization of corecruited proteins by fluorescence microscopy. We recently used this system to show that Atg13 is efficiently recruited to the cytosolic, oligomer-tethered Vac8 site ${ }^{21}$.

We confirmed that ot-Vac $8 \Delta \mathrm{N}-\mathrm{BFP}$ efficiently recruited Atg13-GFP in atg11 $\Delta$ atg19 $v a c 8 \Delta$ cells (Fig. 3c, d). Similarly, Vac8 $\Delta$ N-GFP was efficiently recruited to Atg13-ot-BFP (Supplementary Fig. $4 \mathrm{~b}, \mathrm{c})$. In contrast, GFP-Atg11 did not interact with ot-Vac8 $\triangle$ N-BFP (Fig. 3d, e), nor did ot-Atg11-BFP interact with Vac $8 \Delta$ N-GFP (Supplementary Fig. 4b, c). Similarly, Atg11-GFP did not interact with ot-Vac $8 \Delta \mathrm{N}-\mathrm{BFP}$, however, was efficiently recruited to BFP-Ape1 oligomers (Supplementary Fig. 4d, e). Therefore, in contrast to the Atg13-Vac8 interaction, Atg11 and Vac8 are unable to interact if only one of the binding partners is clustered.

In the wild-type scenario, Atg11 is clustered on the cargo, and Vac8 is spatially confined on the vacuolar membrane, causing a reduced dimensionality of diffusion. The thereby increased effective local concentration of both binding partners could translate the possibly weak individual low affinity interactions into high avidity binding ${ }^{30}$. To test this possibility, we coexpressed ot-Vac8 $\Delta \mathrm{N}-\mathrm{GFP}$ with Atg11-BFP- $\mu \mathrm{NS}$ in atg13

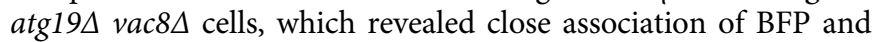
GFP puncta (Fig. 3f, g). These findings suggest that the cargoAtg11 complex is recruited to and anchored at the vacuole by direct interaction of Atg11 with $\mathrm{Vac} 8$ in an avidity-driven manner (Fig. 3h).

Loss of Vac8 prevents PAS maturation during selective autophagy. Our data establish that Vac8 anchors cargo-clustered Atg11 at the vacuole. The early selective PAS formed by the Atg11-cargo and the Atg1 kinase complex subsequently matures via the recruitment of downstream Atg proteins. First, Atg9vesicles and Atg14-containing PI3KC1 associate with the PAS, followed by the independent recruitment of the Atg2-Atg18 
a

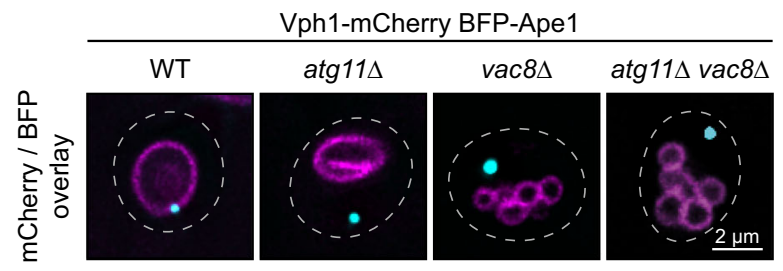

C

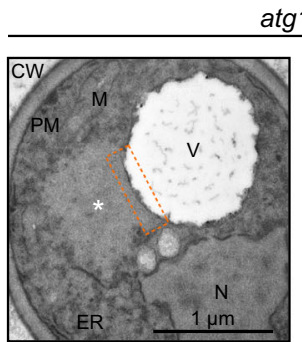

Atg11-GFP- $\mu N S$ $\operatorname{atg} 11 \Delta$

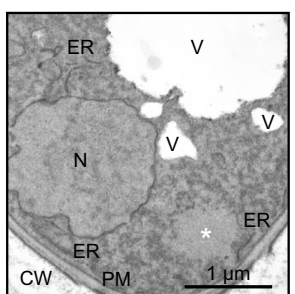

GFP- $\mu N S$ $\operatorname{atg} 11 \Delta$ vac $8 \Delta$

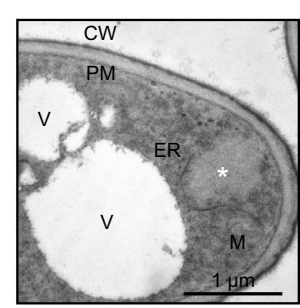

Atg11-GFP- $\mu N S$ b

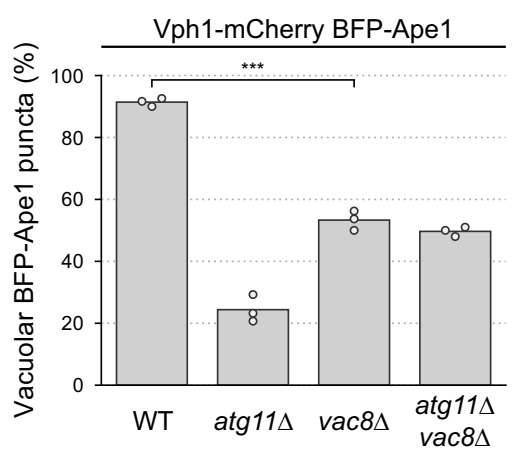

d

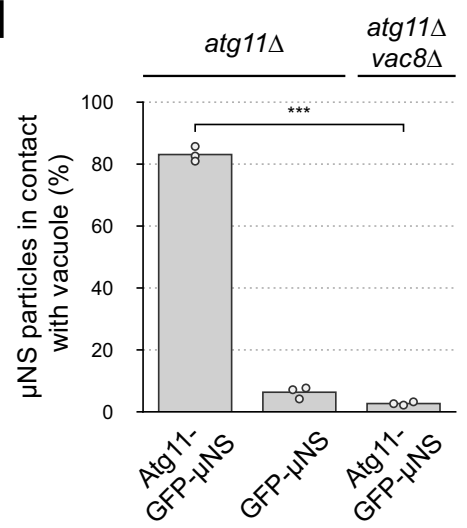

e

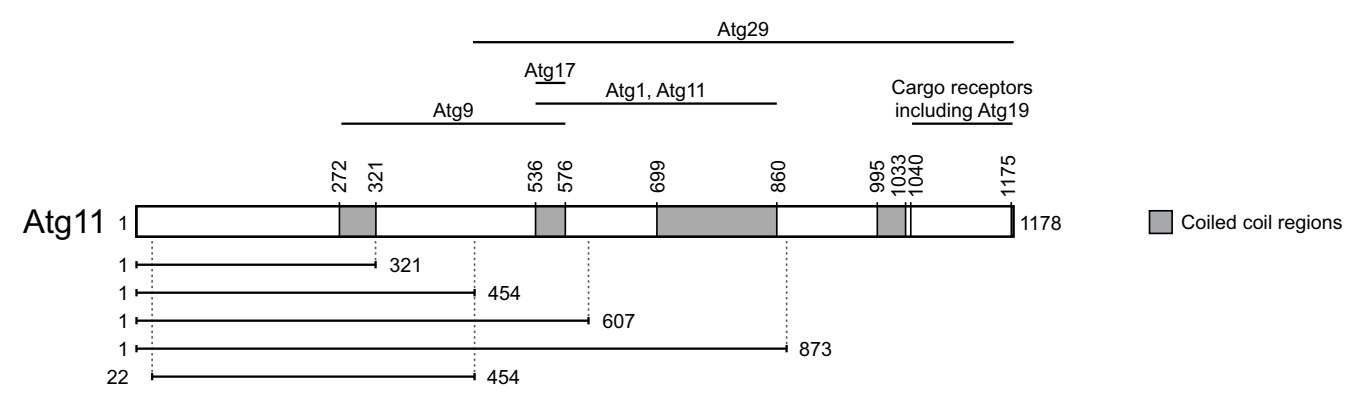

f

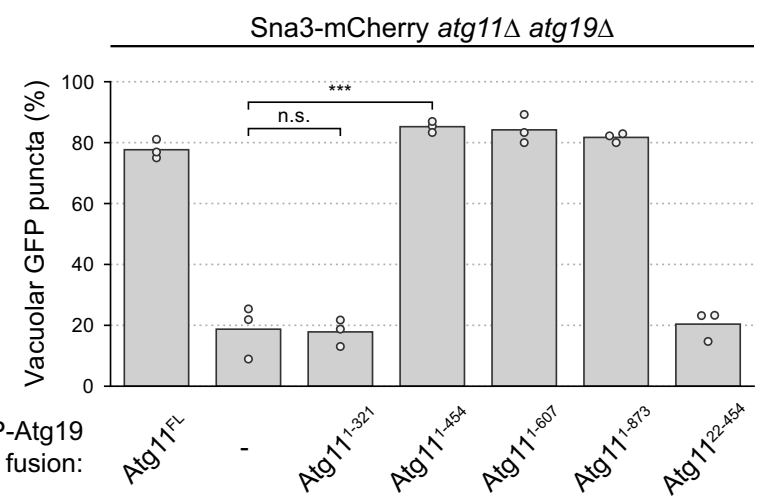

g

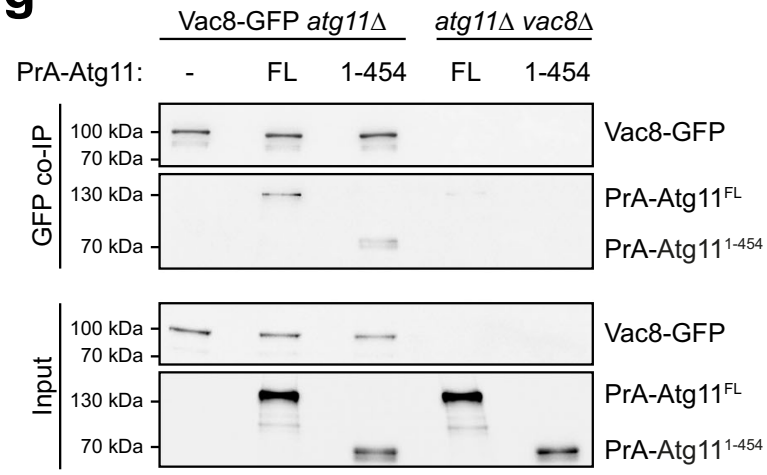

module as well as Atg21, which then results in the recruitment of the Atg12 and Atg8 conjugation systems (Fig. $4 \mathrm{a}^{1}$ ). Whether these downstream events of selective PAS maturation are also affected by Vac8 is unclear.

Under bulk autophagic conditions, the recruitment of the downstream factors Atg2 and Atg8 was not further affected in vac $8 \Delta$ cells. However, vac $8 \Delta$ cells showed an autophagosome-vacuole fusion defect under starvation conditions. Thus, in bulk autophagy, Vac8 is required for initiation of PAS formation and autophagosomevacuole fusion, but not for the maturation of the PAS ${ }^{21}$.

To test if Vac8 influences PAS maturation in selective autophagy, we monitored the formation of GFP-Atg8 puncta, which represent either the PAS or forming or mature autophagosomes. We observed GFP-Atg8 puncta in over $25 \%$ of wild-type 
Fig. 2 Vac8 anchors the selective PAS by interacting with the $\mathbf{N}$-terminus of Atg11. a and $\mathbf{b} \vee$ ph1-4xmCherry wild-type (WT), atg 114 , vac8 4 , and atg11 vac8 $\Delta$ cells containing TagBFP-Ape1 were grown to mid-log phase. a Representative microscopy images showing the overlay of TagBFP-Ape1 and Vph1$4 \times m$ Cherry signal. Dashed lines indicate the contour of individual cells (see also Supplementary Fig. 2a). b The number of vacuolar TagBFP-Ape1 puncta was quantified in three independent experiments. For each strain and replicate at least 27 BFP puncta were analyzed. The values of each replicate (circle) and the mean (bars) were plotted. Statistical analysis using two-tailed unpaired $t$-tests. Significance is indicated with asterisks: ${ }^{\star \star \star} p<0.001,{ }^{\star \star} p<0.01$, ${ }^{\star} p<0.05$, n.s. (not significant) $p>0.05$. Exact numerical values are reported in the source data. $\mathbf{c}$ and $\mathbf{d}$ The indicated strains containing Atg11-GFP- $\mu$ NS or GFP- $\mu$ NS were grown to mid-log phase and processed for electron microscopy as described in "Materials and Methods". c Representative electron micrographs are shown. CW cell wall, PM plasma membrane, M mitochondria, ER endoplasmic reticulum, N nucleus, V vacuole, asterisk Atg11-GFP- $\mu$ NS or GFP- $\mu$ NS particles. The orange dashed box indicates the contact site between Atg11-GFP- $\mu$ NS and the vacuole. $\mathbf{d}$ The percentage of $\mu$ NS particles in contact with the vacuole was quantified in three independent technical replicates. The values of each replicate (circle) and the mean (bars) were plotted. Statistical analysis using two-tailed unpaired $t$-tests. Significance is indicated with asterisks: ${ }^{\star \star \star} p<0.001,{ }^{\star \star} p<0.01,{ }^{\star} p<0.05$, n.s. (not significant) $p>0.05$. Exact numerical values are reported in the source data. e Schematic illustration of Atg11 truncations used in this study. Gray boxes indicate previously mapped

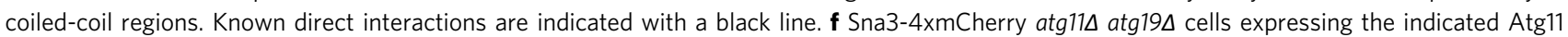

truncations fused to GFP-Atg19 or expressing only GFP-Atg19, were grown to mid-log phase. The number of vacuolar GFP puncta was quantified in three independent experiments. For each strain and replicate at least 28 BFP puncta were analyzed. The values of each replicate (circle) and the mean (bars) were plotted. Representative images are shown in Supplementary Fig. 3a. g Vac8-GFP atg11 cells containing 2xProteinA-Atg11FL, 2xProteinA-Atg111-454 or a control plasmid, or atg $11 \Delta$ vac8 $\Delta$ control cells expressing $2 x$ ProteinA-Atg11 $\mathrm{FL}$ or $2 x$ ProteinA-Atg11 $1-454$ were grown to mid-log phase, followed by glass bead lysis. Vac8-GFP was immunoprecipitated and the amount of precipitated Vac8 and coprecipitated Atg11 was analyzed by anti-GFP and anti-protein A western blotting. One representative experiment out of three independent experiments is shown. FL full length, PrA protein A.

control cells. In contrast, only $8 \%$ of vac $8 \Delta$ cells displayed GFPAtg8 puncta, suggesting that Vac8 is required for PAS maturation and the formation of autophagic membranes (Fig. $4 \mathrm{~b}$ and Supplementary Fig. 5a). Therefore, Vac8 plays an additional role in selective autophagy that is mediated in a Vac8-independent manner in bulk autophagy.

To test which step during PAS maturation requires Vac8 function, we analyzed the formation of PAS puncta containing Atg14 and Atg9. We used an atg8 background, which stalls autophagosome formation and prevents PAS turnover. This allows a precise analysis of PAS formation independent of its turnover, which is therefore directly comparable in different mutant situations. The number of cells containing Atg14-GFP puncta drastically dropped from $50 \%$ in atg $8 \Delta$ or atg1 $\Delta$ atg13 $\Delta$ atg $8 \Delta$ cells to $5 \%$ in atg $8 \Delta$ atg $19 \Delta$ and atg $8 \Delta$ atg $9 \Delta$ control cells, as expected, given that Atg19 is required to initiate selective autophagy and Atg9 is required to recruit Atg14-containing PI3KC1. Importantly, less than $2 \%$ of cells formed Atg14-GFP puncta in atg8 $\operatorname{vac} 8 \Delta$ cells, suggesting that Vac8 is required to recruit Atg14 to the PAS (Fig. 4c, and Supplementary Fig. 5b-d). In contrast, Atg9-GFP vesicles were recruited to BFP-Ape1 puncta with similar frequency in atg $8 \Delta$ and atg $8 \Delta$ vac $8 \Delta$ cells, but not in atg8 $\operatorname{atg} 19 \Delta$ control cells (Fig. $4 \mathrm{~d}$ and Supplementary Fig. 5e). These findings suggest that Atg9 vesicle recruitment to the PAS is independent of Vac8, whereas Atg14 recruitment requires $\mathrm{Vac} 8$ function.

Vac8 recruits the PI3KC1 to the vacuole independent of autophagy function. We noticed that Atg14-GFP localized evenly along the vacuolar membrane in atg19 $\Delta$ cells that lack PAS formation, whereas in vac8 $\Delta$ cells, the vacuolar localization of Atg14-GFP was lost (Fig. 4e and Supplementary Fig. 5f). We therefore speculated that $\mathrm{Vac} 8$ is not only required to recruit Atg14 to the PAS, but is also responsible for Atg14 recruitment to the vacuolar membrane in general. To test this possibility, we analyzed Atg14-GFP membrane association in fractionation experiments. Pgk1 and Tom 20 served as cytosol and membrane markers, respectively. Whereas Atg14-GFP was found both in the cytosol and membrane fraction of wild-type cells, its membrane association was severely reduced in vac8 8 cells. Deletion of ATG11 did not affect Atg14 levels in the membrane fraction, further supporting that the PAS is dispensable for Atg14 membrane association (Fig. 4f). These results suggest that deletion of
VAC8 blocks PAS maturation by inhibiting the recruitment of Atg14 to the vacuole.

Atg14 and Atg38 are part of the autophagy specific PI3KC1, whereas Vps30, Vps15 and Vps34 are subunits of both PI3KC1 and the endocytic PI3K complex $2^{31}$. To test if Vac8 affects vacuolar recruitment of other subunits of the PI3KC1 in addition to Atg14, we analyzed Vps15 localization. Vps15-GFP localized to the vacuolar membrane in wild-type but not in vac8s cells (Fig. $4 \mathrm{~g}$ and Supplementary Fig. $5 \mathrm{~g}$ ). These findings suggest that the entire $\mathrm{PI} 3 \mathrm{KC} 1$ requires $\mathrm{Vac} 8$ for recruitment to the vacuolar membrane.

To test if the PI3KC1 still assembles in the absence of VAC8, we performed co-IP experiments. We found that Vps15-GFP and Vps34-GFP co-immunopurified Atg14-TAP in both wild-type and in vac8 8 cells. The amount of co-immunopurified Atg14 was even higher in vac8 $\Delta$ cells, suggesting that the PI3KC1 assembles independently of its vacuolar membrane association (Fig. 4h).

If Vac8 directly anchors the PI3K complex at the vacuole, as it does clustered Atg11, then ectopic Vac8 should also recruit PI3K complex members. Indeed, clustered ot-Vac $8 \Delta \mathrm{N}-\mathrm{BFP}$ recruited

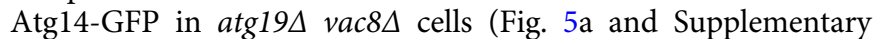
Fig. 6a). To test if the interaction between Atg14 and Vac8 is independent of its interactions with $\operatorname{Atg} 13^{16}$ and Atg11 (Fig. 3a),

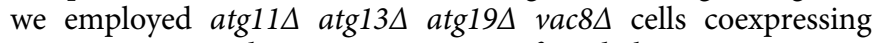
Atg14-ot-BFP and Vac8 $\Delta \mathrm{N}$-GFP. We found that Atg14-ot-BFP efficiently recruited $\mathrm{Vac} 8 \Delta \mathrm{N}$ in both atg19 $v a c 8 \Delta$ and $\operatorname{atg} 11 \Delta$ atg13 $\Delta$ atg $19 \Delta$ vac8 $\Delta$ cells, suggesting that the interaction of the PI3K complex with Vac8 is independent of Atg11 and Atg13 (Supplementary Fig. 6b, c).

From these results, we hypothesized that Atg14 localizes to the vacuole in a Vac8-dependent, but autophagy independent manner. To test this, we coexpressed Atg14-GFP together with Vps30, which is required to stabilize Atg14 ${ }^{31}$, in multipleknockout (MKO) cells that lack 25 Atg proteins ${ }^{32}$. Indeed, Atg14GFP still localized to the vacuolar membrane in the absence of the core autophagy machinery (Supplementary Fig. 6d).

Moreover, we found that Vps15-GFP was recruited to ot-

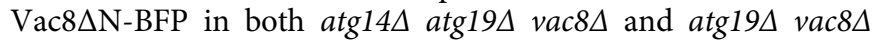
vps $30 \Delta$ cells, suggesting that the interaction of Vac8 and the PI3K complex does not depend on Atg14 and Vps30, and is mediated by Vps15, Vps34 or the Vps15-Vps34 dimer (Fig. 5b and Supplementary Fig. 6e). As vps34 $\Delta$ cells show severely reduced protein levels of Atg14 and Vps15 31 , the effect of VPS34 deletions could not be analyzed in this setup. 
a

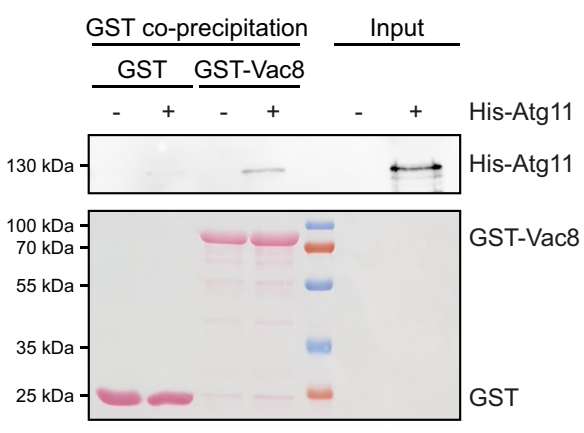

b

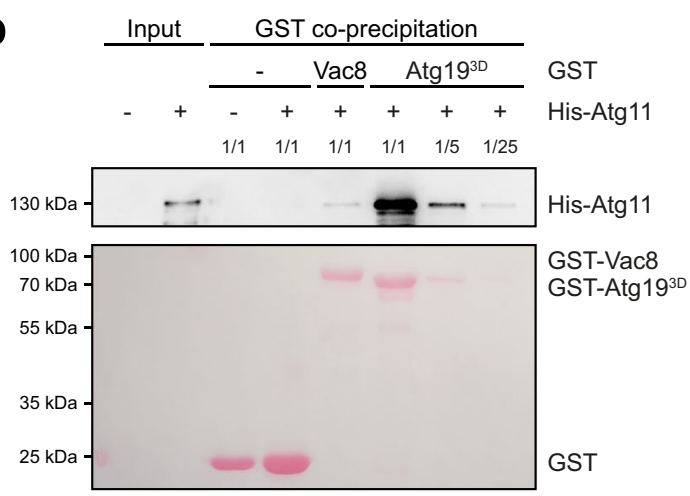

C

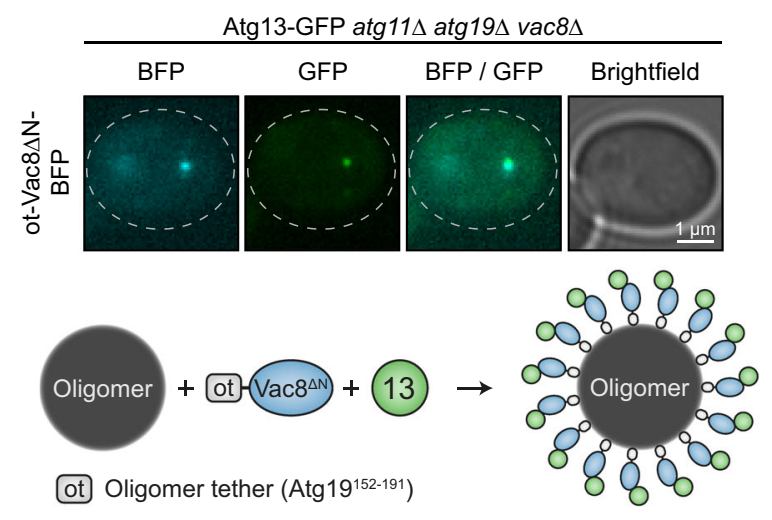

$\mathbf{e}$

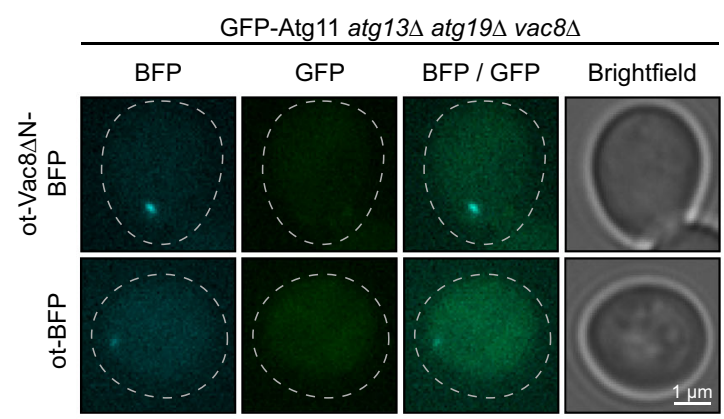

g

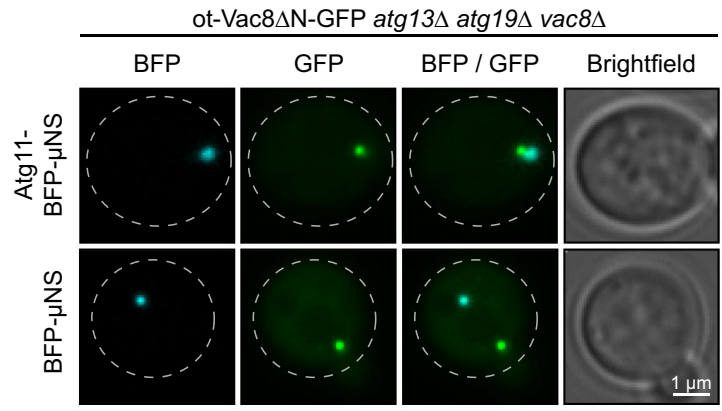

d

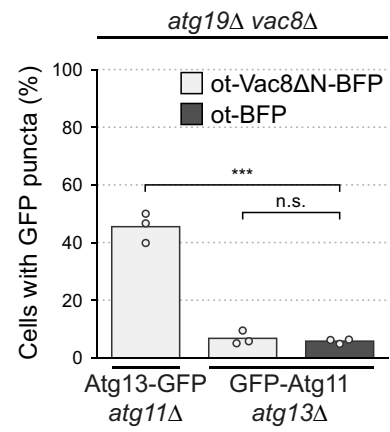

$\mathbf{f}$

ot-Vac8 $\Delta$ N-GFP $\operatorname{atg} 13 \Delta \operatorname{atg} 19 \Delta$ vac $8 \Delta$

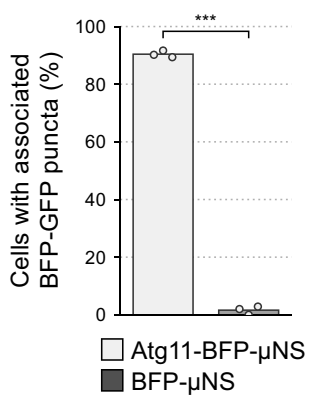

h

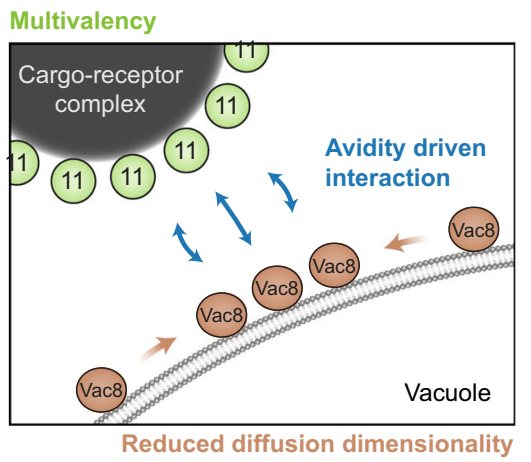

In summary, these findings demonstrate that Vac8 is responsible for the vacuolar recruitment of the PI3K complex via interaction with the Vps15-Vps34 subcomplex. This recruitment does not require Atg14, Atg11, Atg13, nor any other core autophagy protein, thus, PI3KC1 vacuole recruitment is independent of autophagy.
PI3KC1 PAS association requires vacuolar localization. Vac8 independently recruits three autophagy modules to the vacuole: the Atg1-kinase complex via Atg13, the PI3KC1, and the Atg11-cargo. If the main function of Vac8 is to tether PAS factors to the vacuole and thereby facilitate PAS maturation, then artificial tethering of these factors to the vacuole should restore PAS assembly in vac $8 \Delta$ cells. To 
Fig. 3 Vac8-Atg11 interaction is direct and requires clustering. a and b GST, GST-Vac8 or GST-Atg193D (S390D, S391D, and S396D) was expressed in E.coli and bound to Glutathione Sepharose ${ }^{\circledast}$ GSH) beads and further incubated with SF9 insect-cell lysates containing overexpressed 8xHis-Atg11 or control lysates. The amount of coprecipitated 8xHis-Atg11 was monitored by anti-Atg11 western blotting, and the amount of GST or GST-Vac8 bound to GSH beads was monitored by Ponceau S staining. One representative experiment out of three independent experiments is shown. GST glutathione S-transferase. b A 5 times and 25 times dilution of the GST-Atg193D co-IP was loaded to compare the amount of coprecipitated 8xHis-Atg11 between GST-

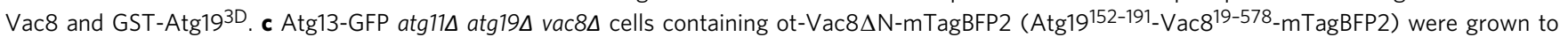
mid-log phase. The recruitment of Atg13-GFP to ot-Vac8 $\Delta$ N-BFP puncta was monitored by fluorescence microscopy. Dashed lines indicate the contour of individual cells. ot oligomer tether. d Quantification of $\mathbf{c}$ and $\mathbf{e}$. The percentage of cells with GFP puncta was quantified in three independent biological replicates. For each condition and replicate at least 100 cells were analyzed. The values of each replicate (circle) and the mean (bars) were plotted. Statistical analysis using two-tailed unpaired $t$-tests. Significance is indicated with asterisks: ${ }^{\star \star \star} p<0.001,{ }^{\star \star} p<0.01,{ }^{\star} p<0.05$, n.s. (not significant) $p>0.05$. Exact numerical values are reported in the source data. e GFP-Atg11 atg13 $\Delta$ atg19 $\Delta$ vac8 $\Delta$ cells containing ot-Vac8 $\Delta N-m T a g B F P 2$ or ot-mTagBFP2 were grown to mid-log phase. The recruitment of GFP-Atg11 to ot-Vac8 N -BFP puncta was monitored. Dashed lines indicate the contour of individual cells. $\mathbf{f}$ and $\mathbf{g}$ atg $13 \Delta$ atg $19 \Delta$ vac8 $\Delta$ cells containing ot-Vac8 $\Delta$ N-GFP and Atg11-mTagBFP2- $\mu$ NS or mTagBFP2- $\mu$ NS were grown to mid-log phase. Colocalization of GFP and BFP puncta was analyzed. $\mathbf{f}$ The percentage of cells with associated BFP and GFP puncta was quantified in three independent biological replicates. For each condition and replicate at least 35 cells containing BFP and GFP puncta were analyzed. The values of each replicate (circle) and the mean (bars) were plotted. Statistical analysis using two-tailed unpaired $t$-tests. Significance is indicated with asterisks: ${ }^{\star \star \star} p<0.001,{ }^{\star \star} p<0.01,{ }^{\star} p<0.05, n . s$. (not significant) $p>0.05$. Exact numerical values are reported in the source data. $\mathbf{g}$ Representative fluorescence microscopy images of $\mathbf{f}$ are shown. Dashed lines indicate the contour of individual cells. $\mathbf{h}$ Schematic illustration of the Vac8-Atg11 interaction: Atg11 clusters on cargo-receptor complexes, whereas Vac8 is confined at the vacuolar membrane. These local concentrations of Vac8 and Atg11 allow the otherwise low-affinity interactions between Vac8 and Atg11 to be stabilized by avidity.

test this possibility, we tethered Atg11 and Atg14 to the vacuole in vac8 $\Delta$ cells. Specifically, we fused Atg11 to the vacuolar transmembrane protein Vph1 ( $\mathrm{vt}^{1}-\mathrm{mScarlet}$-Atg11, $\mathrm{vt}^{1}=$ vacuole tether 1$)$ and Atg14 to the transmembrane domain of the vacuolar membrane protein Pho8 (Atg14-GFP-vt ${ }^{2}$ ).

We found that mScarlet-Atg11 clustered on the cargo but was unable to associate with the vacuole in vac8 $\Delta$ cells, as expected. In contrast, $\mathrm{vt}^{1}$-mScarlet-Atg11 displayed stable vacuolar localization in vac8 $\Delta$ cells, which did not require cargo binding via Atg19 (Supplementary Fig. 7a). Similarly, Atg14-GFP-vt ${ }^{2}$, but not Atg14-GFP, showed stable vacuole association in the absence of Vac8 (Supplementary Fig. 7b).

To investigate if vacuole tethering of Atg11 and Atg14 restores PAS formation in vac8s cells, we tethered Atg11, Atg14 or both to vacuoles in BFP-Ape1 containing vac8 8 cells. We found that vacuole recruitment of BFP-Ape1 oligomers was restored in vac8 $\Delta$ cells expressing $\mathrm{vt}^{1}$-mScarlet-Atg11 (Fig. 5 c, column 2). This recruitment was dependent on Atg19, demonstrating that $\mathrm{vt}^{1}$-mScarlet-Atg11 recruits Ape1-Atg19 oligomers (Fig. 5c, column 7). However, cytosolic Atg14-GFP was not recruited to puncta containing BFP-Ape1 and $\mathrm{vt}^{1}$-mScarlet-Atg11 (Fig. $5 c, d$, column 2), suggesting that the vacuolar localization of PI3KC1 is required for its association with PAS structures. Intriguingly, Atg14-GFP-vt ${ }^{2}$ restored PAS formation of cytosolic mScarletAtg11 and BFP-Ape1 to $60 \%$ of wild-type levels, suggesting that vacuole-localized PI3KC1 can recruit cargo-Atg11 complexes in the absence of Vac8 (Fig. 5d, column 4). Importantly, coexpression of $\mathrm{vt}^{1}-\mathrm{mScarlet}-\mathrm{Atg} 11$ and Atg14-GFP-vt ${ }^{2}$ in vac8s cells restored PAS formation (Fig. 5d, compare columns 1 and 5), which required the Ape1 cargo receptor Atg19 and Atg9 (Fig. 5d, columns 6 and 7), further supporting that Atg9 is required for PI3KC1 to associate with the PAS (Fig. 5c, d). Although Atg14 was dispensable for the initial recruitment of the Atg11-cargo complex to the vacuolar membrane (Fig. 1a), the PI3KC1 might play a role in stabilizing the PAS-vacuole connection during phagophore formation (Fig. 5c, d, column 4).

These findings suggest that vacuolar localization of the PI3KC1 and Atg11-cargo is sufficient to restore their efficient association in the absence of Vac8 (Fig. 5e). In particular, local confinement of PI3KC1 to the vacuole is a prerequisite for its association with the PAS, suggesting that this interaction depends on avidity, similar to Vac8-Atg11. In summary, these findings support that
PAS formation is an avidity-driven process guided by multiple low-affinity interactions, coordinated by $\mathrm{Vac} 8$ at the vacuole.

Reconstitution of PAS formation at the nucleus is sufficient to drive selective autophagy. To further substantiate that Vac8 acts as a spatial regulator of PAS formation during selective autophagy, we reconstituted this process ectopically in vivo. We used the nuclear membrane as an ectopic site in the cell, as it represents a single and defined membranous structure. In the absence of vacuolar Vac8, such as $\operatorname{Vac} 8 \Delta \mathrm{N}$, nucleus-vacuole junctions (NVJs) are lost. Therefore, nuclei do not contact the vacuole in vac $8 \Delta$ mutants or $\operatorname{Vac} 8 \Delta \mathrm{N}$ mutant cells ${ }^{14,33,34}$. To tether Vac8 to the nucleus, we fused cytosolic Vac $8 \Delta \mathrm{N}$ to the N-terminal, 125 amino acid, transmembrane domain of the nuclear outer membrane protein Nvj1 (nt-mScarlet-Vac $8 \Delta \mathrm{N}$; nt = nucleus tether). We observed nt-mScarlet-Vac $8 \Delta \mathrm{N}$ distribution along the outer nuclear membrane in vac8 8 cells (Fig. 6a).

To test if nt-mScarlet-Vac $8 \Delta \mathrm{N}$ can recruit GFP-Atg11-cargo complexes, we quantified the nuclear association of GFP-Atg11 puncta. Indeed, $60 \%$ of GFP-Atg11 puncta were recruited to the nucleus in vac $8 \Delta$ cells expressing nt-mScarlet-Vac $8 \Delta \mathrm{N}$, but only $10 \%$ of GFP-Atg11 puncta were at the nucleus in vac $8 \Delta$ cells expressing nt-mScarlet (Fig. 6b and Supplementary Fig. 8a). Similar to the redistribution of vacuolar Vac8-mCherry to the vacuolar contact sites of Atg11-GFP- $\mu$ NS (Fig. 1f), also nuclear nt-mScarlet-Vac $8 \Delta \mathrm{N}$ enriched at the nuclear contact sites of Atg11-GFP- $\mu$ NS. (Supplementary Fig. $8 \mathrm{~b}$ ). These data suggest that nuclear-localized nt-Vac8 $\Delta \mathrm{N}$ efficiently recruits the Atg11cargo complex.

Since Vac8 recruits PI3KC1 to the vacuole (Fig. 4e, g), we reasoned that nt-mScarlet-Vac $8 \Delta \mathrm{N}$ should recruit PI3KC1 to the nucleus. We monitored Vps15-GFP localization in vac $8 \Delta$ cells expressing nt-mScarlet-Vac $8 \Delta \mathrm{N}$ and found that $60 \%$ of cells showed Vps15-GFP recruitment to the nucleus, whereas vac8 $\Delta$ cells expressing nt-mScarlet lacked nuclear localization of Vps15GFP (Fig. 6c and Supplementary Fig. 8c).

Functional PI3KC1 produces PI3P at the PAS and thereby allows the recruitment of the PI3P-dependent autophagy factors, the Atg2-Atg18 complex and Atg21 (Fig. 4a). To test if PI3P production at this ectopic PAS is functional, we monitored the formation of Atg2-GFP puncta, which is a well-established readout for the production of PI3P at the PAS and the subsequent 
a

Hierarchy of selective PAS assembly

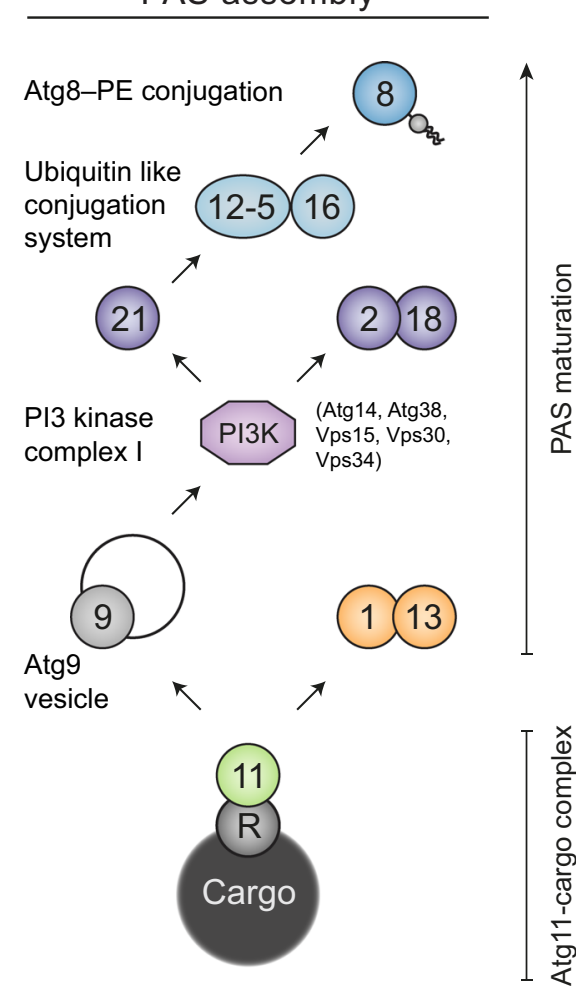

b

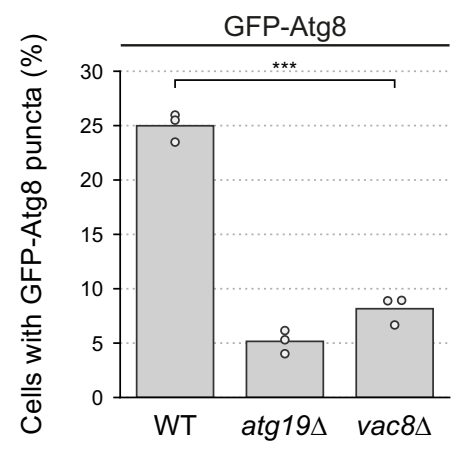

d

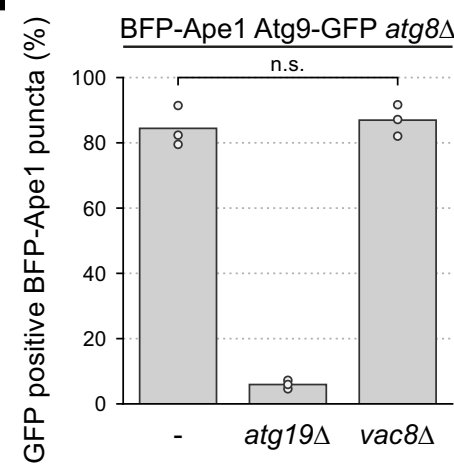

C

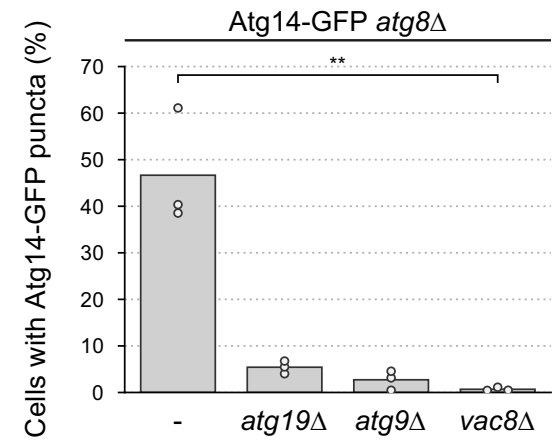

$\mathbf{e}$

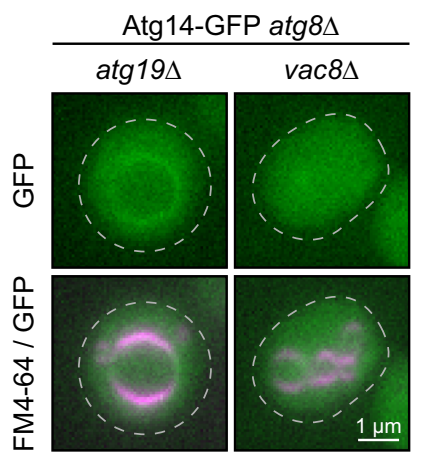

g

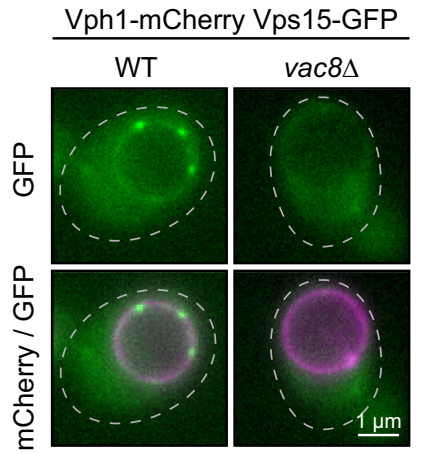

h

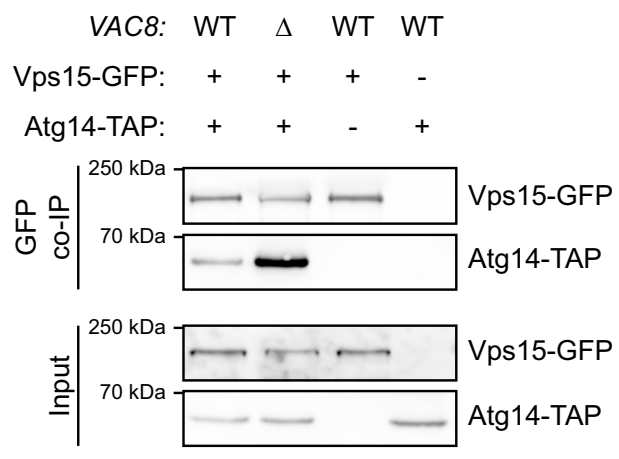

recruitment of the Atg2-Atg18 complex ${ }^{35,36}$. Strikingly, Atg2GFP formed puncta in vac8 4 atg $8 \Delta$ cells expressing nt-mScarletVac $8 \Delta \mathrm{N}$, but not in $v a c 8 \Delta$ atg $14 \Delta$ cells, vac8 $\Delta$ atg11 $\Delta$ cells or those expressing nt-mScarlet, suggesting that Atg2-GFP associates with the ectopic PAS in a PI3P-dependent manner (Fig. 6d, and Supplementary Fig. 8d-f).

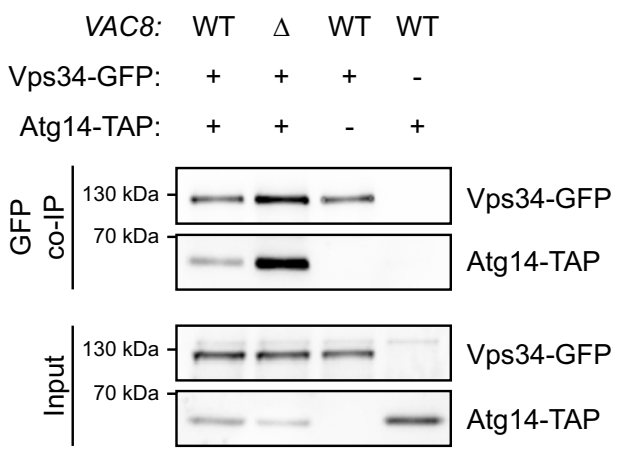

The final step in PAS maturation is the recruitment of Atg8, which depends on Atg21 and thus PI3P production (Fig. 4a). Similar to Atg2-GFP, GFP-Atg8 puncta also formed in vac8 $\Delta$ cells expressing nt-mScarlet-Vac $8 \Delta \mathrm{N}$ in an Atg14-dependent manner (Fig. 6e and Supplementary Fig. 9a). Thus, ectopic Vac8 stimulates complete PAS maturation at a nonvacuolar membrane. 
Fig. 4 Loss of Vac8 prevents PAS and vacuole recruitment of the PI3K complex. a Model of hierarchical recruitment of selective PAS factors based on the literature. See also for details. Circles containing numbers correspond to the respective Atg proteins. PE, phosphatidylethanolamine. $\mathbf{b}$ The indicated strains containing GFP-Atg8 were grown to mid-log phase. The percentage of cells with GFP-Atg8 puncta was analyzed in three independent biological replicates. For each condition and replicate at least 100 cells were analyzed. The values of each replicate (circle) and the mean (bars) were plotted. Statistical analysis using two-tailed unpaired $t$-tests. Significance is indicated with asterisks: ${ }^{\star \star \star} p<0.001,{ }^{\star \star} p<0.01,{ }^{\star} p<0.05$, n.s. (not significant) $p>0.05$. Exact numerical values are reported in the source data. See Supplementary Fig. 5a for representative fluorescence microscopy images. WT wildtype. c The indicated strains containing Atg14-3xGFP were grown to mid-log phase. The percentage of cells with Atg14-3xGFP puncta was analyzed in three independent biological replicates. For each condition and replicate at least 100 cells were analyzed. The values of each replicate (circle) and the mean (bars) were plotted. Statistical analysis using two-tailed unpaired $t$-tests. Significance is indicated with asterisks: ${ }^{\star \star \star} p<0.001,{ }^{\star \star} p<0.01,{ }^{\star} p<0.05$, n.s. (not significant) $p>0.05$. Exact numerical values are reported in the source data. See Supplementary Fig. $5 b$ for representative fluorescence microscopy images. d The indicated Atg9-GFP strains containing mTagBFP2-Ape1 were grown to mid-log phase. The percentage of GFP positive BFP-Ape1 puncta was analyzed in three independent biological replicates. For each condition and replicate at least 60 BFP puncta were analyzed. The values of each replicate (circle) and the mean (bars) were plotted. Statistical analysis using two-tailed unpaired $t$-tests. Significance is indicated with asterisks: ${ }^{\star \star \star} p<0.001$, ${ }^{\star \star} p<0.01$, ${ }^{\star} p<0.05$, n.s. (not significant) $p>0.05$. Exact numerical values are reported in the source data. See Supplementary Fig. 5 e for representative fluorescence

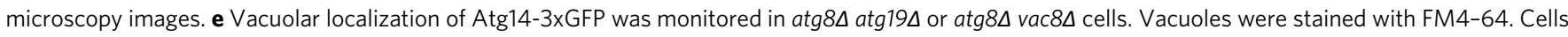
were grown to mid-log phase. Representative fluorescence microscopy images of one out of two independent experiments are shown. Dashed lines indicate the contour of individual cells (see also Supplementary Fig. 5f). $\mathbf{f}$ The indicated Atg14-3xGFP strains were subjected to cell lyses and subsequent fractionation. Supernatant and pellet fractions were separated at 20,000 $\times g$, and the distribution of Atg14-3xGFP in different fractions was monitored by anti-GFP western blotting. Wild-type cells without GFP-tagged Atg14 were used as a control. Pgk1 was used as a cytosolic marker, Tom20 was used as membrane marker. One representative experiment out of two independent experiments is shown. In, input; S, supernatant; $\mathrm{P}$, pellet fraction. $\mathbf{g}$ Vacuolar localization of Vps15-GFP was monitored in Vph1-4xmCherry wild-type and vac8D cells. Cells were grown to mid-log phase. Representative fluorescence microscopy images of one out of two independent experiments are shown. Dashed lines indicate the contour of individual cells (see also Supplementary Fig. 5g). $\mathbf{h}$ The indicated Vps15-GFP, Vps34-GFP or wild-type strains containing Atg14-TAP or an empty plasmid were grown to mid-log phase. Cell extracts were subjected to GFP immunoprecipitation using GFP-trap affinity resin, and the amount of precipitated protein and coprecipitated Atg14-TAP was analyzed by anti-GFP and anti-protein A western blotting. One representative experiment out of three independent experiments is shown.

Selective autophagosome formation is blocked in vac8s cells compared with wild-type cells ${ }^{16}$. Our results suggest that nt$\mathrm{mScarlet}-\mathrm{Vac} 8 \Delta \mathrm{N}$ can completely reconstitute PAS maturation at the nucleus in vac8 8 cells. To determine if this ectopic PAS could restore autophagosome formation, we followed GFP-Atg8 puncta formation in the presence or absence of the Rab GTPase YPT7. ypt7D mutants are deficient in autophagosome-vacuole fusion and thus accumulate mature autophagosomes ${ }^{37}$. Indeed, we observed accumulation of GFP-Atg8 puncta in fusion deficient GFP-Atg8 nt-mScarlet-Vac $8 \Delta \mathrm{N} v a c 8 \Delta y p t 7 \Delta$ cells, suggesting that autophagosomes are formed and fuse with the vacuole in Ypt7 containing GFP-Atg8 nt-mScarlet-Vac $8 \Delta \mathrm{N}$ vac8 $\Delta$ cells (Supplementary Fig. 9b, c). Interestingly, redirecting PAS formation to the nuclear membrane in vac8 Y YPT7 cells already resulted in an increase of GFP-Atg8 puncta compared with VAC8 wild-type cells, indicating a reduced fusion efficiency of ectopically formed autophagosomes.

To confirm that autophagosomes are also turned over in these reconstituted cells, we monitored vacuolar uptake of BFP-Ape1. Excitingly, over $40 \%$ of vac $8 \Delta$ cells expressing nt-mScarletVac $8 \Delta \mathrm{N}$ showed diffuse vacuolar BFP-Ape1 signal, similar to wild-type cells, and this rescue required the cargo receptor Atg19 (Fig. 7a, b). Vacuolar Ape1 processing was also restored in vac8 $\Delta$ cells expressing nt-mScarlet-Vac $8 \Delta \mathrm{N}$ (Fig. 7c). Thus, reconstitution of an ectopic PAS at the nucleus is sufficient to restore selective autophagy function.

Together, these findings show that Vac8 acts to coordinate PAS formation at the vacuole by recruiting and assembling multiple Atg proteins into the PAS at this membrane site. PAS assembly is governed by avidity, which contributes to the spatiotemporal control of PAS formation during selective autophagy and in succession drives pathway progression (Fig. $7 \mathrm{~d}$ ).

\section{Discussion}

In this study, we developed a synthetic reconstitution approach in live cells to discover the mechanisms underlying PAS assembly. In particular, we dissected the protein interactions and their dependencies that mediate PAS subcellular localization and maturation.
We find that autophagosome formation during selective autophagy in budding yeast is spatially organized by multiple aviditydriven interactions, coordinated at the vacuole by Vac8.

Avidity is an important regulatory feature of many biological systems. The concept of avidity explains how multivalent systems translate individual low affinity interactions into much higher functional affinities. Although each individual interaction may be readily broken, it will likely be restored because the additional interactions prevent structures from diffusing away, thus maintaining a high effective concentration of binding partners. The high avidity of IgM pentamers, for example, renders them particularly efficient at binding antigens present at low levels, but also prevents aberrant interactions ${ }^{30}$.

Whereas the concept of avidity has emerged as an important biological feature in extracellular systems, it has been mostly ignored in intracellular regulation ${ }^{30}$. The initiation of selective autophagy represents an intracellular process depending on high avidity interactions. Cargo-dependent clustering of autophagy factors provides multivalency of binding partners. In addition, the restriction of early autophagy factors to a specific membrane site leads to a reduced dimensionality of diffusion and therefore increases their local concentration. These features of PAS formation ensure a robust and decisive assembly process based on binding thresholds, and could make selective autophagy largely irreversible once initiated.

The initiation of bulk autophagy is regulated by the assembly of a higher order oligomeric structure by autophagy scaffold proteins ${ }^{38}$. This assembly provides an increase in the local concentration of early autophagy factors, promoting multivalent interactions, similar to clustering on the surface of selective cargo. It has recently been suggested that this assembly structure also undergoes transient phase separation ${ }^{19}$. In this context phase separation might fulfill an analogous function to clustering on the cargo $^{39-41}$, providing avidity by limiting diffusion of phase separated factors.

Vac8 interacts with Atg13, which recruits and confines the Atg1 kinase to the vacuolar membrane. This allows Atg1 to bind Atg11-bound to cargo complexes, resulting in clustering-induced 
a

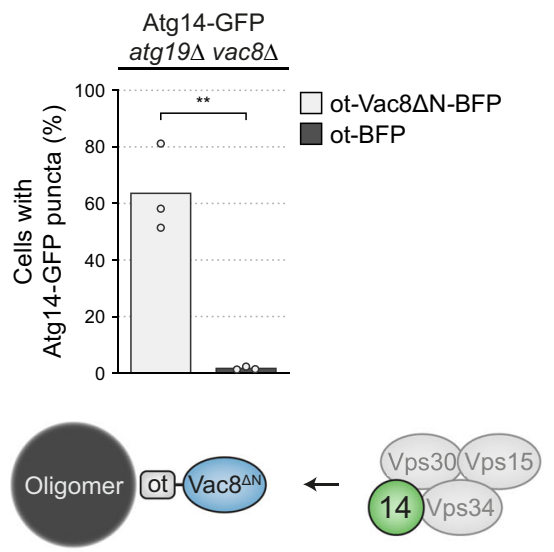

b

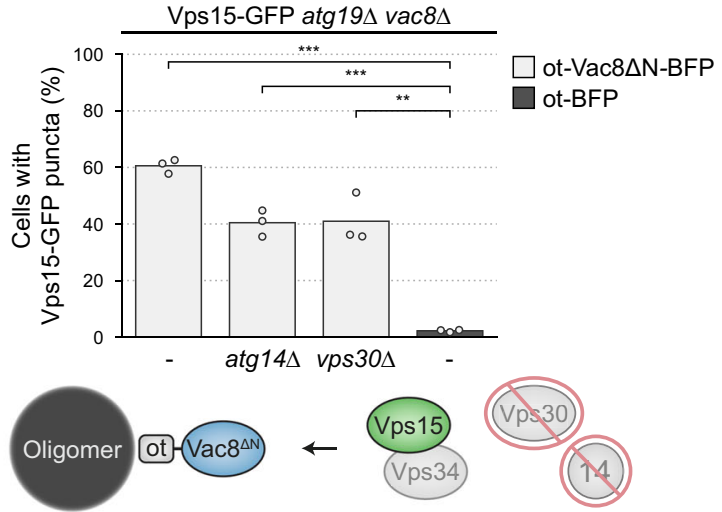

C

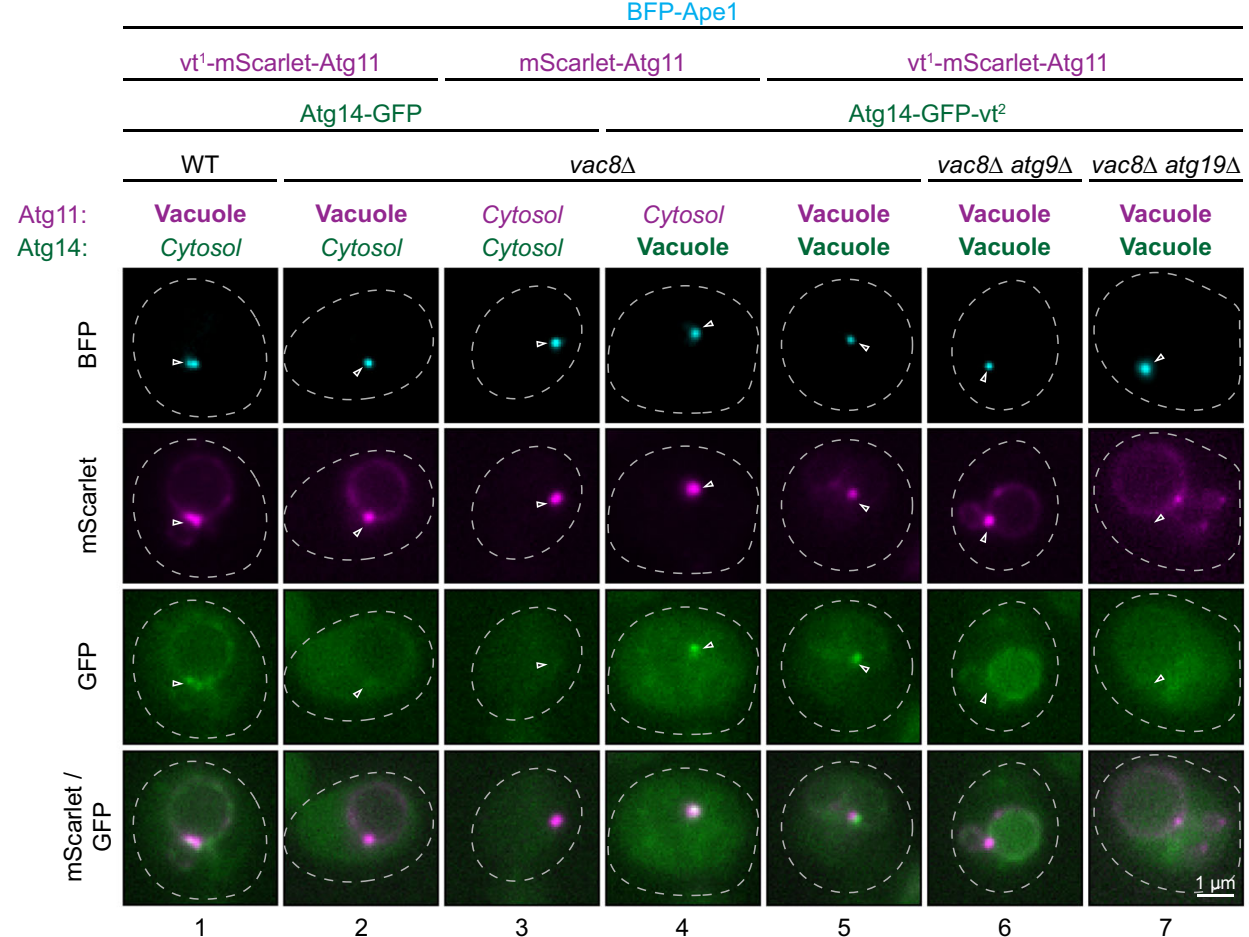

d
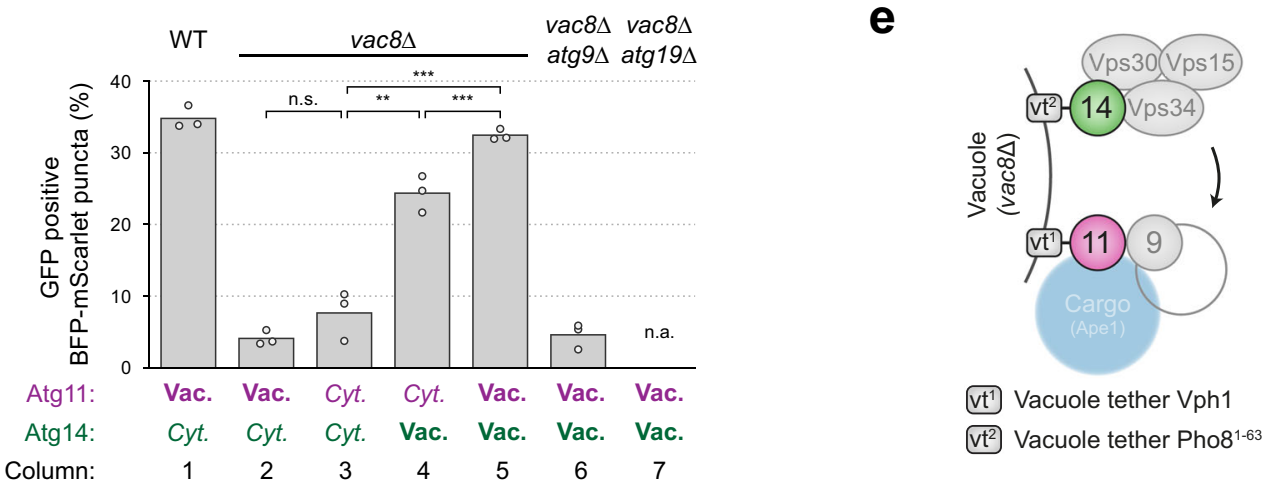

trans-autophosphorylation and local kinase activation at the vacuole $^{7,8}$. Here we find that $\mathrm{Vac} 8$ also confines the PI3KC1 to the vacuolar membrane to promote a similar avidity-dependent interaction with the Atg11-cargo complex bound to Atg9 vesicles. Vac8 also directly interacts with Atg11 to recruit the autophagy cargo complex to the vacuole. Stable interaction between Vac8 and Atg11 requires clustering on both sides, demonstrating that the tethering of the PAS to the vacuole is also achieved via highavidity interactions. Interestingly, all three Vac8-dependent interactions are independent of each other, suggesting a parallel assembly of the PAS at the vacuolar membrane, rather than a linear hierarchy (Fig. 7d).

This mode of PAS formation displays striking similarities with the assembly of the mammalian autophagy-initiation complex at 


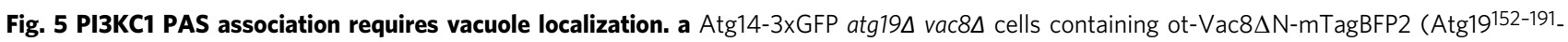
Vac8 ${ }^{19-578}$-mTagBFP2) or ot-mTagBFP2 were grown to mid-log phase. The recruitment of Atg14-3xGFP to BFP puncta was analyzed by fluorescence microscopy. The percentage of cells with GFP puncta was analyzed in three independent biological replicates. For each condition and replicate at least 100 cells were analyzed. The values of each replicate (circle) and the mean (bars) were plotted. A schematic of the experimental setup is shown below. Statistical analysis using two-tailed unpaired $t$-tests. Significance is indicated with asterisks: ${ }^{\star \star \star} p<0.001,{ }^{\star \star} p<0.01,{ }^{\star} p<0.05$, n.s. (not significant) $p>0.05$. Exact numerical values are reported in the source data. See Supplementary Fig. 6a for representative fluorescence microscopy images. ot, oligomer tether. $\mathbf{b}$ The indicated Vps15-GFP strains containing ot-Vac8 $\mathrm{N}$-mTagBFP2 or ot-mTagBFP2 were grown to mid-log phase. The recruitment of Vps15-GFP to BFP puncta was analyzed by fluorescence microscopy. The percentage of cells with GFP puncta was analyzed in three independent biological replicates. For each condition and replicate at least 100 cells were analyzed. The values of each replicate (circle) and the mean (bars) were plotted. A schematic of the experimental setup is shown below. Statistical analysis using two-tailed unpaired $t$-tests. Significance is indicated with asterisks:

${ }^{\star \star \star} p<0.001,{ }^{\star \star} p<0.01,{ }^{\star} p<0.05$, n.s. (not significant) $p>0.05$. Exact numerical values are reported in the source data. See Supplementary Fig. 6e for representative fluorescence microscopy images. c and $\mathbf{d}$ The indicated mTagBFP2-Ape1 strains containing Vph1-mScarlet-Atg11 or mScarlet-Atg11 and Atg14-GFP or Atg14-GFP-Pho8 ${ }^{1-63}$ were grown to mid-log phase. c Representative fluorescence microscopy images are shown. Dashed lines indicate the contour of individual cells, white arrows indicate the position of mScarlet-Atg11 positive mTagBFP2-Ape1 puncta. Note that the BFP and mScarlet channels were individually contrasted due to differences in brightness. $\mathbf{d}$ The percentage of GFP positive BFP-mScarlet puncta was quantified in three independent biological replicates. For each condition and replicate at least 50 BFP-mScarlet puncta were analyzed. The values of each replicate (circle) and the mean (bars) were plotted. Statistical analysis using two-tailed unpaired $t$-tests. Significance is indicated with asterisks: ${ }^{\star \star \star} p<0.001,{ }^{\star \star} p<0.01,{ }^{\star} p<0.05$, n.s. (not significant) $p>0.05$. Exact numerical values are reported in the source data. WT, wild-type. e Schematic of the experimental setup used in $\mathbf{c}$ and $\mathbf{d}$. vac. vacuole, cyt. cytosol, n.a. not applicable, vt ${ }^{1}$ vacuole tether $V_{p h 1}$ vt $^{2}$ vacuole tether $\mathrm{Pho}^{1-63}$.

the ER membrane. First, FIP200 (Atg11 in yeast) associates with ER membranes, localizing to phosphatidylinositol synthase (PIS)enriched ER subdomains. Downstream factors are subsequently recruited, initiating phagophore formation at this site ${ }^{11}$. FIP200 and these downstream factors are visible as discrete puncta at the ER in fluorescence microscopy, rather than as a homogeneous reticular ER staining ${ }^{11,42}$, indicating that assembly happens at discrete ER subdomains. Similarly in yeast, cargo-bound Atg11 at the vacuolar membrane guides the recruitment of downstream factors to this specific location, leading to the formation of the PAS. In mammals, FIP200 recruits the ULK1 kinase complex, while in yeast Atg11 recruits the Atg1 kinase complex. In both cases, this results in local clustering and activation of the kinase, which is required for autophagy progression. FIP200 associates with the ER independently of PI3KC3-C1, ATG9A and other members of the ULK1 complex. Moreover, PI3KC3-C1 is recruited to the ER likely independently of FIP200 and other autophagy players, whereas FIP200 is required to recruit PI3KC3$\mathrm{C} 1$ to the PIS-enriched ER subdomains ${ }^{11,43-45}$. This is also analogous to Atg11 in yeast, which is recruited to the vacuole independently of the PI3KC1, Atg9 and the Atg1 complex, whereas the PI3KC1-PAS association depends on Atg11. Furthermore, both yeast PI3KC1 and mammalian PI3KC3-C1 require Atg9/ATG9A-positive vesicles to be recruited to the PAS (Fig. $4 \mathrm{c}^{42}$ ). We propose that PAS formation in mammals, like in yeast, involves avidity-dependent interactions that are driven by clustering at the autophagic cargo and confinement on ER membranes. If a central coordinator such as Vac8 also exists in mammals, if this role could be mediated by PIS enrichment, or if independent ER recruitment mechanisms exist for FIP200 and the PI3KC3-C1, remains unknown. During the revision of this paper, ARMC3 has been suggested as a potential homolog of $\mathrm{Vac}^{46}$.

Clustered Atg11- $\mu$ NS induced massive relocalization of Vac8 on the vacuolar membrane to the contact site of Atg11- $\mu$ NS and exclusion of Vph1 and possibly further vacuolar proteins (Fig. 1f, $\mathrm{g}^{22}$ ). This rearrangement of vacuolar proteins demonstrates their mobility in the 2-dimensional membrane plane and is similar to rearrangements happening at organelle-organelle contact sites, such as Nvj1 enrichment at the nucleus-vacuole junctions or the ERMES (ER-mitochondria encounter structure) at ERmitochondria contact sites ${ }^{47-49}$. As Vac8 acts as the central hub in organizing the PAS, one can speculate that the mobility of membrane-associated proteins is required for recruiting multiple complexes into one location at the vacuole. We successfully reconstituted autophagosome formation at a heterologous site, by tethering a cytosolic Vac8 mutant to the outer nuclear membrane. The vacuole per se seems therefore dispensable for this process. If tethering Vac8 to a rigid structure such as a protein oligomer would interfere with its ability to promote PAS formation, and if the fluidity of a membrane is required, will be interesting to address in future studies.

Most autophagy proteins localize to multiple positions at or all along the phagophore during expansion, whereas the PI3KC1 and Atg13 remain only at the junction between the phagophore and the vacuolar membrane ${ }^{50}$. As Vac8 recruits both these factors to the vacuole, $\mathrm{Vac} 8$ is likely responsible for their retention at this site. The production of PI3P on the phagophore thus might be spatially restricted to the region on the phagophore that is tethered to the vacuole, from where PI3P is then distributed to the entire phagophore.

In contrast to selective autophagy, deletion of VAC8 did not abrogate Atg2 and Atg8 puncta formation in bulk autophagy ${ }^{21}$. Since the recruitment of both of these proteins depends on PI3P, $\mathrm{Vac} 8$ is likely not strictly required for $\mathrm{PI} 3 \mathrm{KC1}$ recruitment to the bulk autophagy PAS. Also, in contrast to selective autophagy, VAC8 deletion cells still form autophagosomes under starvation, however at a substantially reduced rate and smaller in size. This reduced size could stem from a failure in efficient PI3P production at the bulk PAS in vac8 4 cells $^{21}$. How exactly PAS association of the PI3KC1 is regulated in bulk autophagy and if its confinement to the vacuolar membrane is also involved, remains to be determined in more detail. Vac8 in bulk autophagy is furthermore required for the efficient fusion of autophagosomes with the vacuole ${ }^{21}$. Our findings indicate that Vac8 might also be involved in autophagosome-vacuole fusion during selective autophagy (Supplementary Fig. 9b, c).

Clustering of cargo receptors has been recognized as an important regulatory mechanism for selective autophagy. Activation of the Atg1/ULK1 kinase requires their auto-phosphorylation, which is achieved by their clustering on cargo complexes $^{8,51,52}$. The requirement of cargo receptor clustering has also been attributed to phagophore growth, where avidity is required to ensure the stable interaction of cargo complexes with growing autophagic membranes ${ }^{53-56}$. Here clustering of cargo receptors and membrane confinement of Atg8 on the phagophore allow the individual low affinity interactions of receptors with Atg8 to be stabilized by avidity. 
a

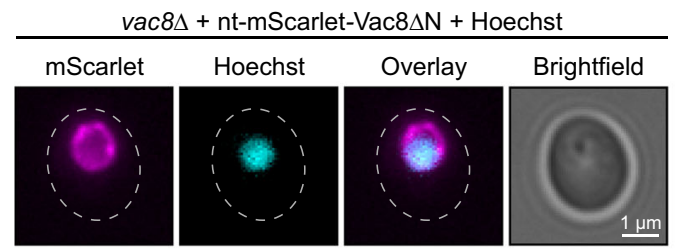

b



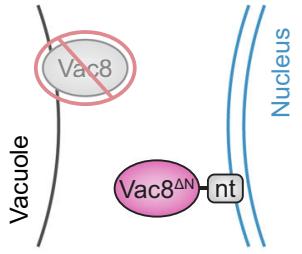

nt Nucleus tether $\left(\mathrm{Nvj} 1^{1-125}\right)$

\section{C}
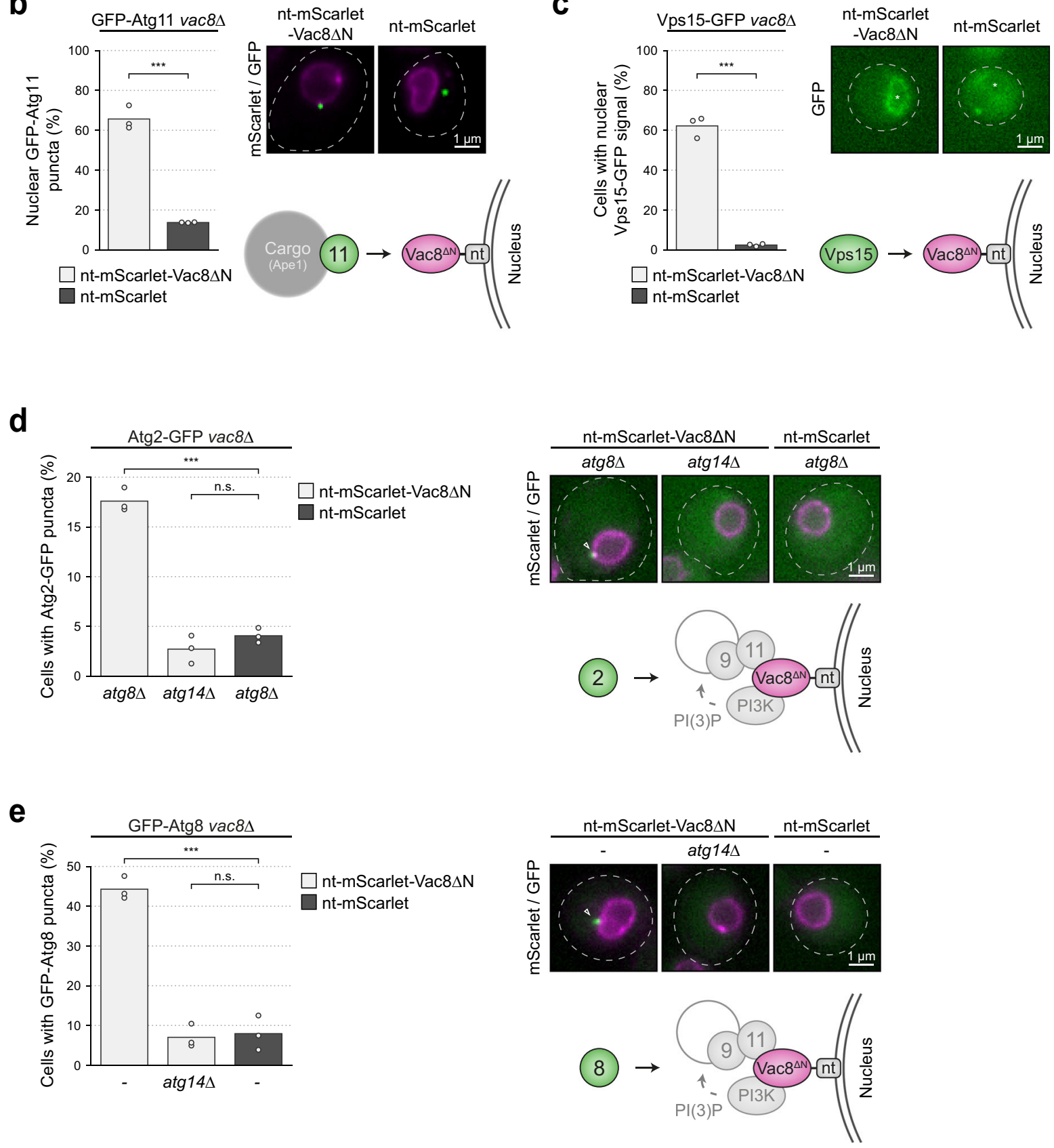

Our findings, however, indicate that this initial cargo receptor clustering not only aids phagophore binding and Atg1/ULK1 kinase activation, but serves to initiate PAS formation by controlling cargo localization and recruitment of the PI3KC1. Following the initial clustering of Atg11 on cargo-receptor complexes and its recruitment to the vacuole, PAS assembly

might proceed in a self-organized manner by avidity-driven assembly steps. The recruitment of the Atg machinery in this manner and the many interactions taking place at a confined space support a robust and well controlled assembly process and prevent arbitrary autophagy induction or aberrant autophagosome formation. 
Fig. 6 Reconstitution of PAS formation at the nucleus is sufficient for complete PAS assembly. a Vph1-GFP vac8 $\Delta$ cells containing nt-mScarlet-Vac8 $\Delta N$ (Nvj1 ${ }^{1-125}$-mScarlet-Vac8 ${ }^{19-578}$ ) were analyzed. The nucleus was stained with Hoechst. Representative fluorescence microscopy images are shown. Dashed lines indicate the contour of individual cells. A schematic of the experimental setup is shown on the right. One representative experiment out of two independent experiments is shown. nt, nucleus tether (Nvj1-125). b GFP-Atg11 vac8 $\Delta$ cells containing nt-mScarlet-Vac8 $8 \mathrm{~N}$ or nt-mScarlet were grown to mid-log phase. The recruitment of GFP-Atg11 puncta to the nuclear membrane was analyzed. The percentage of nuclear GFP puncta was quantified in three independent biological replicates. For each condition and replicate at least 100 GFP puncta were analyzed. The values of each replicate (circle) and the mean (bars) were plotted. Representative fluorescence microscopy images displaying an overlay of the mScarlet and GFP signal and a schematic of the experimental setup are shown on the right. Statistical analysis using two-tailed unpaired $t$-tests. Significance is indicated with asterisks: ${ }^{\star \star \star} p<0.001$, ${ }^{* \star} p<0.01,{ }^{*} p<0.05$, n.s. (not significant) $p>0.05$. Exact numerical values are reported in the source data. See also Supplementary Fig. 8a. c Vps15-GFP vac $8 \Delta$ cells containing $\mathrm{nt}-\mathrm{mScarlet-Vac} 8 \Delta \mathrm{N}$ or $\mathrm{nt}-\mathrm{mScarlet}$ were grown to mid-log phase. The recruitment of Vps15-GFP to the nuclear membrane was analyzed. The percentage of cells with nuclear GFP signal was quantified in three independent biological replicates. For each condition and replicate at least 100 cells were analyzed. The values of each replicate (circle) and the mean (bars) were plotted. Representative fluorescence microscopy images of the GFP signal and a schematic of the experimental setup are shown on the right. White asterisk indicates the position of the nucleus. Statistical analysis using twotailed unpaired $t$-tests. Significance is indicated with asterisks: ${ }^{\star \star \star} p<0.001,{ }^{\star \star} p<0.01,{ }^{\star} p<0.05$, n.s. (not significant) $p>0.05$. Exact numerical values are reported in the source data. See also Supplementary Fig. 8c. d The indicated Atg2-GFP strains containing nt-mScarlet-Vac8 $\Delta \mathrm{N}$ or nt-mScarlet were grown to mid-log phase. The formation of Atg2-GFP puncta was analyzed. The percentage of cells with GFP puncta was quantified in three independent biological replicates. For each condition and replicate at least 100 cells were analyzed. The values of each replicate (circle) and the mean (bars) were plotted. Representative fluorescence microscopy images displaying an overlay of the mScarlet and GFP signal and a schematic of the experimental setup are shown on the right. A white arrow indicates a GFP punctum. Statistical analysis using two-tailed unpaired $t$-tests. Significance is indicated with asterisks: ${ }^{\star \star \star} p<0.001,{ }^{\star \star} p<0.01,{ }^{\star} p<0.05$, n.s. (not significant) $p>0.05$. Exact numerical values are reported in the source data. See also Supplementary Fig. 8d. e The indicated GFP-Atg8 strains containing nt-mScarlet-Vac8 $8 \mathrm{~N}$ or nt-mScarlet were grown to mid-log phase. Experiments were quantified and presented as described in d. See also Supplementary Fig. 9a.

\begin{abstract}
Methods
Yeast strains and plasmids. Yeast strains are listed in Supplementary Data 1. Plasmids are listed in Supplementary Data 2, and plasmid sequence maps are available in Supplementary Data 3. Yeast genomic mutations were integrated by homologous recombination; genomic insertions (tagging) were performed according to 57,58 and multiple deletions or mutations were generated by PCR knockout, mating, and dissection. GFP-ATG8 and GFP-ATG11 containing strains were generated by crossing with yTB281 and yTB2838,28, respectively, which had been generated by seamless tagging ${ }^{59}$. yDH459 (mTagBFP2-APE1), yDH480 and yDH481 were generated by homologous recombination of $m$ TagBFP2-APE1:LEU2 from pDH4 into the leu2 $\Delta 0$ locus of BY4741. yDH459 was used to cross further strains.
\end{abstract}

Growth conditions. Yeast cells were grown in synthetic medium (SD, $0.17 \%$ yeast nitrogen base, $0.5 \%$ ammonium sulfate, $2 \%$ glucose, and amino acids as required) or rich medium (YPD, $1 \%$ yeast extract, $2 \%$ peptone, and $2 \%$ glucose) to mid-log phase. Yeast cultures were incubated with shaking $(220 \mathrm{rpm})$ at $30^{\circ} \mathrm{C}$.

Antibodies. The following primary antibodies were used in this study. Antisera were diluted in $1 \mathrm{x}$ PBS pH 7.4 containing 5\% milk powder. Mouse monoclonal anti-GFP (1:100, clone 2B6, Merck, cat \# MABC1689), rabbit polyclonal PAP antibody (for protein A and TAP detection, 1:3000, Sigma-Aldrich, cat \# P1291), mouse monoclonal anti-Pgk1 antibody (1:30,000, clone 22C5D8, Invitrogen, cat \# 459250), rabbit polyclonal anti-Atg1 antibody (1:15,000, Daniel Klionsky, University of Michigan, USA), rabbit polyclonal anti-Tom 20 antibody (1:500, Nora Voegtle, University of Freiburg, Germany), rabbit polyclonal anti-Atg19 antibody (1:5000, Sascha Martens, University of Vienna, Austria), rabbit polyclonal antiApel antibody (1:15,000, Claudine Kraft, University of Freiburg, Germany), mouse monoclonal anti-Atg11 antibody (1:500, clone 6F4-G4, Claudine Kraft, University of Freiburg, Germany), mouse monoclonal anti-Atg17 antibody (1:50, clone 4D3E8, Claudine Kraft, University of Freiburg, Germany), and mouse monoclonal antiAtg29 antibody (1:25, clone 1C4-D5, Claudine Kraft, University of Freiburg, Germany). The anti-Atg17 and anti-Atg29 antibodies were generated at the Max Perutz Laboratories Monoclonal Antibody Facility by immunizing Balb/c mice with recombinant proteins containing full length Atg17-Atg31-Atg29 purified from Sf9 insect cells. Splenocytes were fused with X63-Ag8.653 myeloma cells, and hybridoma cells were established in HAT selection medium. Hybridoma supernatants were screened for the presence of specific antibodies by immunoblotting, and positive candidates were monoclonalized. The maintenance of mice and the experimental procedures have been conducted according to the Austrian Animal Experiments Act and have been approved by the Austrian Federal Ministry of Science and Research BMWFW-66.009/0211-WF/V3b/2015, and the animal experiments ethics committee of the Medical University of Vienna.

Standard biochemical assays. Yeast cell culture or yeast extracts and fractions thereof were precipitated with $7 \%$ trichloroacetic acid (TCA) for $30 \mathrm{~min}$ on ice or overnight at $-20^{\circ} \mathrm{C}$. Precipitated proteins were pelleted at $16,000 \times \mathrm{g}$ for $15 \mathrm{~min}$ at $4^{\circ} \mathrm{C}$, washed with $1 \mathrm{ml}$ of acetone, air-dried, resuspended in urea loading buffer (120 mM Tris- $\mathrm{HCl} \mathrm{pH} 6.8,5 \%$ glycerol, $8 \mathrm{M}$ urea, $143 \mathrm{mM} \beta$-mercaptoethanol, and
$8 \%$ SDS), boiled, and analyzed by SDS-PAGE. Protein extracts were transferred to nitrocellulose membranes, and proteins were detected by immunoblotting, using the ECL detection system with HRP coupled secondary antibodies. Uncropped western blots are provided in the source data.

Yeast-extract preparation and immunoprecipitation. For preparation of yeast extract by freezer milling, cells were harvested by filtration on a $90 \mathrm{~mm}$ glass filter (SterliTech) using a nitrocellulose membrane with a pore size of $0.45 \mu \mathrm{m}$ followed by freezing in liquid nitrogen. Cells were milled in a cryogenic grinder (SPEX Freezer Mill 6875, SPEX SamplePrep), using five rounds of $3 \mathrm{~min}$ breakage at 15 cycles per second and 2 min of cooling, and the powder was stored at $-80^{\circ} \mathrm{C}$. Freezer milled yeast powder was resuspended in RLB + buffer (1x PBS pH 7.4, 10\% glycerol, $0.5 \%$ Tween-20, $1 \mathrm{mM}$ sodium fluoride, $20 \mathrm{mM} \beta$-glycerol, $1 \mathrm{mM}$ PMSF, $1 \mathrm{mM}$ sodium vanadate and protease inhibitor cocktail [Roche]) and the cell extract was cleared by centrifugation twice at $5000 \times$ for $10 \mathrm{~min}$ at $4{ }^{\circ} \mathrm{C}$ and the supernatant was transferred to a new microfuge tube each time (Supplementary Fig. 3d).

For preparation of yeast extract by glass bead beating, cells were harvested by centrifugation at $3000 \times \mathrm{g}$ for $5 \mathrm{~min}$ at $\mathrm{RT}$, and resuspended in $1 \mathrm{x}$ PBS, $\mathrm{pH} 7.4,2 \%$ glucose. Cells were pelleted at $3000 \times \mathrm{g}$ for $5 \mathrm{~min}$ at RT, and resuspended in $\mathrm{RLB}+$ buffer. Cells were lysed by the addition of glass beads and vortexing for $6 \mathrm{~min}$ at $4{ }^{\circ} \mathrm{C}$. The cell extract was cleared by centrifugation at $573 \times \mathrm{g}$ for $5 \mathrm{~min}$ at $4^{\circ} \mathrm{C}$ (Figs. $2 \mathrm{~g}, 4 \mathrm{~h}$, and Supplementary Fig. 3c).

For immunoprecipitation, the protein concentration of cell extracts was adjusted to $20 \mu \mathrm{g} / \mu \mathrm{l}$ in RLB + buffer. For protein A immunoprecipitation, magnetic Dynabeads ${ }^{\mathrm{TM}} \mathrm{M}-270$ Epoxy (Invitrogen) were coupled with IgG from rabbit serum (Sigma). For GFP immunoprecipitation magnetic GFP-Trap ${ }^{\Phi}$ Dynabeads $^{\text {TM }}$ (Chromotek) were used. The magnetic beads were incubated with cell extract for $1 \mathrm{~h}$ rotating at $4{ }^{\circ} \mathrm{C}$ and washed three times with RLB + buffer. The magnetic beads were resuspended in urea loading buffer, boiled and analyzed by SDS-PAGE and western blotting.

Cell fractionation. In total, $50 \mathrm{OD}_{600}$ units (one $\mathrm{OD}_{600}$ unit corresponds to $1 \mathrm{ml}$ of yeast culture with an $\mathrm{OD}_{600}$ of 1 ) of yeast culture were pelleted at $3000 \times \mathrm{g}$ for $5 \mathrm{~min}$ at RT, resuspended in DTT buffer $(100 \mathrm{mM}$ Tris- $\mathrm{HCl} \mathrm{pH} 9.4$ and $10 \mathrm{mM}$ DTT), and incubated for $15 \mathrm{~min}$ at $30^{\circ} \mathrm{C}$. Cells were pelleted at $3000 \times \mathrm{g}$ for $5 \mathrm{~min}$ at RT, resuspended in SP buffer $(0.25 \%$ YPD, $1 \mathrm{M}$ sorbitol, $50 \mathrm{mM}$ potassium phosphate $\mathrm{pH} 7.5$, and $1 \mathrm{mM}$ DTT), and spheroplasted by lyticase $(100 \mathrm{U} / \mathrm{ml})$ treatment for $30 \mathrm{~min}$ at $30^{\circ} \mathrm{C}$. Spheroplasts were pelleted at $1500 \times \mathrm{g}$ for $5 \mathrm{~min}$ at $4{ }^{\circ} \mathrm{C}$ and washed with PS1000 buffer (1 M sorbitol, $10 \mathrm{mM}$ PIPES-KOH pH 6.8, and complete ${ }^{\mathrm{TM}}$ protease inhibitor cocktail [Roche]). Spheroplasts were pelleted at $1500 \times \mathrm{g}$ for $5 \mathrm{~min}$ at $4{ }^{\circ} \mathrm{C}$, resuspended in PS200 buffer $(200 \mathrm{mM}$ sorbitol, $10 \mathrm{mM}$ PIPES-KOH pH6.8, and complete ${ }^{\mathrm{TM}}$ protease inhibitor cocktail [Roche]) and incubated for $15 \mathrm{~min}$ at $4{ }^{\circ} \mathrm{C}$. Spheroplasts were lysed on ice by pressing through a $22 \mathrm{G}$ needle 15 times. The lysate was cleared by centrifugation at $500 \times \mathrm{g}$ for $5 \mathrm{~min}$ at $4{ }^{\circ} \mathrm{C}$ and transfer of the supernatant to a new microfuge tube until no pellet was observed. To separate the cytosolic from the membrane fraction, the cleared lysate was spun at $20,000 \times \mathrm{g}$ for $20 \mathrm{~min}$ at $4{ }^{\circ} \mathrm{C}$. The supernatant was transferred in a new Eppendorf tube and the pellet was resuspended in PS200 buffer. The supernatant 
a

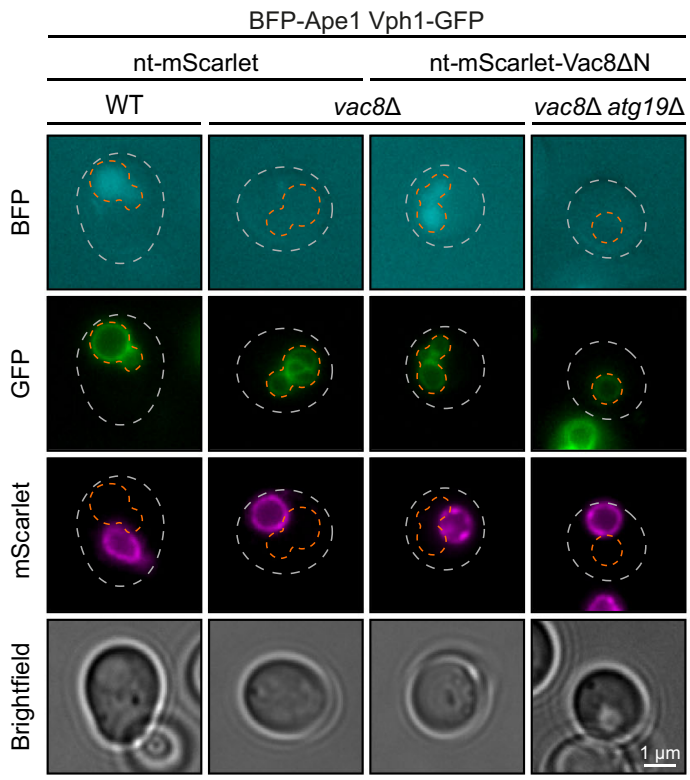

b

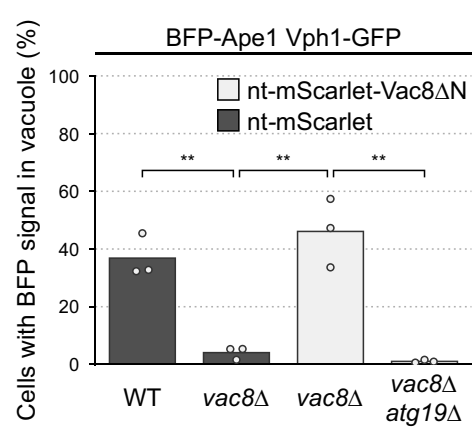

C

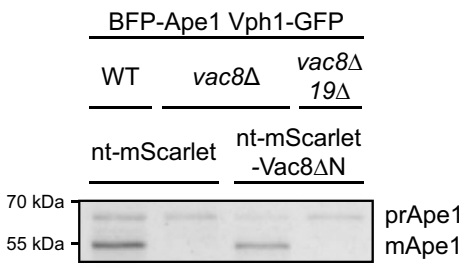

d

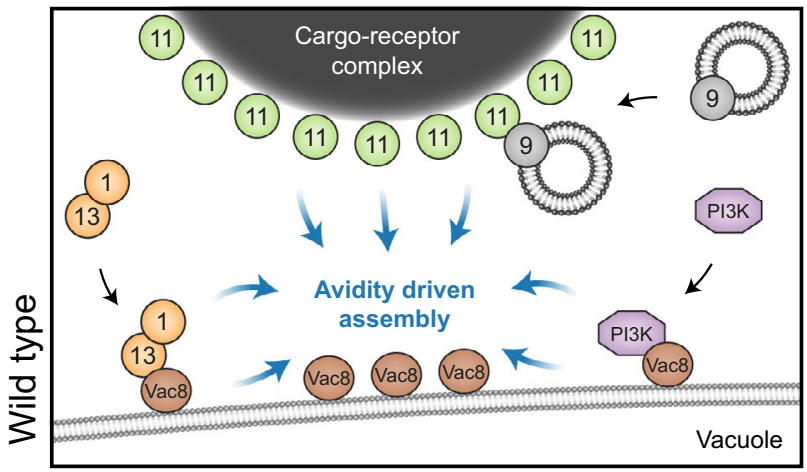

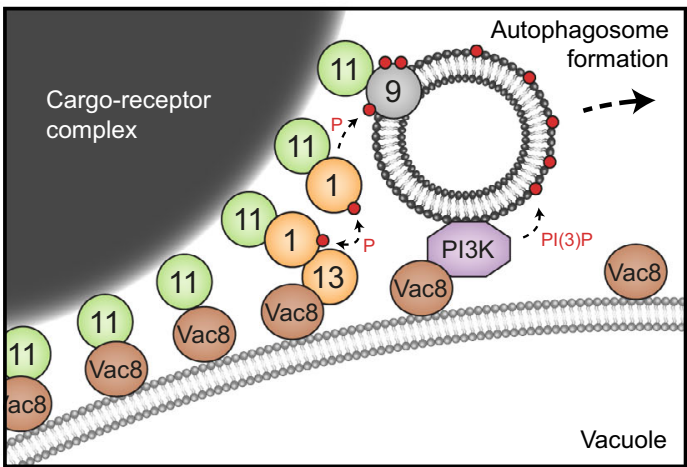
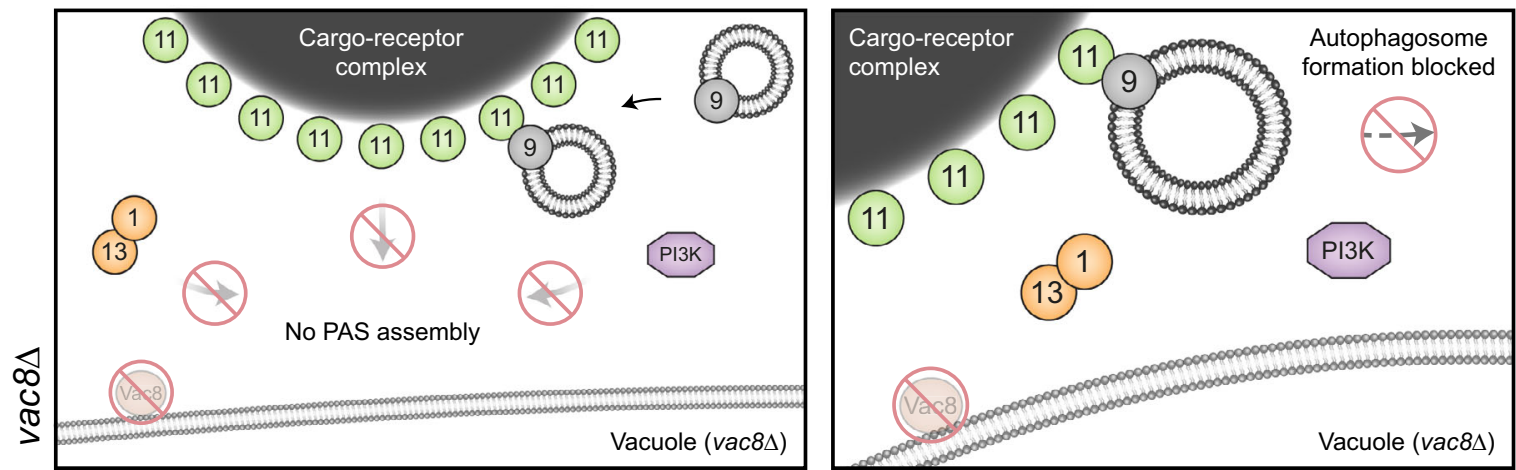

was cleared by centrifugation at $20,000 \times \mathrm{g}$ at $4^{\circ} \mathrm{C}$ for $20 \mathrm{~min}$ and transferred to a new microfuge tube. The pellet fraction was spun at $20,000 \times \mathrm{g}$ at $4{ }^{\circ} \mathrm{C}$ for $20 \mathrm{~min}$ and the pellet was resuspended in PS200 buffer. Cleared supernatant and pellet fractions were TCA precipitated and analyzed by SDS-PAGE and western blotting.

Yeast two-hybrid $(\mathbf{Y} 2 \mathrm{H})$ assay. For $\mathrm{Y} 2 \mathrm{H}$ experiments Atg11 ${ }^{\mathrm{FL}}$-GAD or Atg1 11-454-GAD and LexA-Vac8 fusion constructs were expressed in the S.cerevisiae $\mathrm{L} 40 \mathrm{Y} 2 \mathrm{H}$ reporter strain. In total, $5 \mathrm{OD}_{600}$ units of yeast culture were pelleted at $3000 \times \mathrm{g}$ for $5 \mathrm{~min}$ at RT, resuspended in $30 \mu \mathrm{l}$ of $1 \mathrm{x}$ PBS pH 7.4 and frozen in liquid nitrogen. Cells were thawed at RT and mixed with $100 \mu$ of reaction buffer ( $2 \%$ 5-bromo-chloro-3-indolyl- $\beta$-D-galactosidase in dimethylformamide, $10 \%$ SDS, 1x PBS pH 7.4 and $0.05 \% \beta$-mercaptoethanol), transferred to 96 -well plates, and incubated in the dark at RT. Pictures were taken at several time points within $24 \mathrm{~h}$.

Protein expression and in vitro-binding assay. 8xHIS-Atg11 was expressed from baculovirus-infected Sf9 Spodoptera frugiperda insect cells (Expression Systems, cat \# 94-001 F). The Atg11 ORF was and subcloned with an N-terminal 8xHIS tag into a pLIB library vector ${ }^{60}$. The recombinant bacmid carrying 8xHIS-Atg11 was 
Fig. 7 Vac8 coordinates PAS maturation and defines the site of autophagosome formation. $\mathbf{a}$, $\mathbf{b}$, and $\mathbf{c}$ The indicated Vph1-GFP mTagBFP2-Ape1 strains containing nt-mScarlet-Vac8 $\Delta \mathrm{N}$ or nt-mScarlet were grown to mid-log phase. nt, nucleus tether $\left(\mathrm{Nvj1} 1^{1-125}\right)$. a The transport of mTagBFP2-Ape1 to the vacuole was analyzed. Representative microscopy images are shown. Dashed gray lines indicate the contour of individual cells, dashed orange lines indicate the vacuole. Note that the GFP and mScarlet channels were individually contrasted due to differences in brightness. $\mathbf{b}$ The percentage of cells with vacuolar BFP signal was quantified in three independent biological replicates. For each condition and replicate at least 100 cells were analyzed. The values of each replicate (circle) and the mean (bars) were plotted. Statistical analysis using two-tailed unpaired $t$-tests. Significance is indicated with asterisks: ${ }^{\star * \star} p<0.001,{ }^{\star \star} p<0.01,{ }^{\star} p<0.05$, n.s. (not significant) $p>0.05$. Exact numerical values are reported in the source data. $c$ Cell extracts were prepared by TCA precipitation and Ape1 processing was monitored by anti-Ape1 western blotting. WT wild-type, prApe1 precursor form of Ape1, mApe1 mature form of Ape1. d Vac8 acts as a vacuolar assembly hub by recruiting Atg11-bound cargo-receptor complexes, the Atg1 kinase complex and the PI3KC1 to the vacuole, facilitating their interaction and PAS formation at this site. Stable interaction between Vac 8 and Atg11 depends on avidity, achieved by the local concentration of Atg11 on the cargo and the confinement of $\mathrm{Vac} 8$ at the vacuole. Independent of the cargo the Atg1 kinase complex and PI3KC1 are also recruited to the vacuole by Vac8. Assembly of these three Vac8-interacting complexes into the PAS is then further facilitated by avidity driven interactions: The confinement of the Atg1 kinase complex on the vacuolar membrane allows its stable binding to Atg11-bound cargo, whereas vacuolar confinement of Atg14 facilitates its interaction with Atg9 vesicles that are clustered on Atg11-bound cargo. Atg1 recruitment to cargo-bound Atg11 results in clusteringinduced Atg1 trans-autophosphorylation and kinase activation at the PAS. Atg9 phosphorylation by Atg1 and PI3P production by the PI3KC1 at this site then promote PAS maturation and ultimately the formation of an autophagosome. In the absence of Vac8, the PAS cannot assemble and autophagosomes do not form. Circles containing numbers correspond to the respective Atg proteins. PI3K PI3 kinase complex I, P protein phosphorylation, $\mathrm{PI}(3) \mathrm{P}$ phosphatidylinositol 3-phosphate.

assembled in DH10EMBacY E. coli strain (Geneva Biotech). For 8xHIS-Atg11 expression Sf 9 cells were grown in 11 of ESF 921 Insect Cell Culture Medium (Expression Systems) supplemented with penicillin and streptomycin at $27^{\circ} \mathrm{C}$ to $1 \times 10^{6}$ cells $/ \mathrm{ml}$, infected by the addition of $1 \mathrm{ml}$ of $\mathrm{V} 1$ virus, and grown for 4 days at $27^{\circ} \mathrm{C}$. Cells were pelleted at $500 \times \mathrm{g}$ for $10 \mathrm{~min}$ at RT, washed with $1 \mathrm{x}$ PBS pH 7.4 , and the pellet was frozen in liquid nitrogen and stored at $-80^{\circ} \mathrm{C}$. Cell pellets were resuspended in lysis buffer ( $500 \mathrm{mM}$ Tris $\mathrm{pH} 7.4,1.5 \mathrm{M} \mathrm{KCl}, 50 \mathrm{mM} \mathrm{MgCl}$, $10 \%$ glycerol, $5 \mathrm{mM} \beta$-mercaptoethanol, $1 \mathrm{mM}$ PMSF, $0.1 \%$ Triton X-100, $10 \mathrm{mM}$ imidazole, complete ${ }^{\mathrm{TM}}$ protease inhibitor cocktail [Roche]), Benzonase ${ }^{\circledR}(25 \mathrm{U} / \mathrm{ml}$; MERCK) was added, and cells were lysed by 12 passes using a Dounce homogenizer (WHEATON ${ }^{\oplus}$ Dounce Tissue Grinder). Two freeze-thaw cycles were performed. Cell lysates were frozen in liquid nitrogen followed by thawing at $4{ }^{\circ} \mathrm{C}$, with additional Benzonase ${ }^{\circledast}(25 \mathrm{U} / \mathrm{ml}$, MERCK) being added before each freezing step. Cell lysates were cleared three times by centrifugation at $30,000 \times \mathrm{g}$ for $10 \mathrm{~min}$ at $4{ }^{\circ} \mathrm{C}$ and the supernatant was transferred to a new microfuge tube each time.

GST, GST-Vac8, and GST-Atg193D fusion constructs were expressed from pGEX-4T-1 in E. coli BL21(DE3). Cells were grown in lysogeny broth (LB) medium supplemented with ampicillin at $37^{\circ} \mathrm{C}$, until an $\mathrm{OD}_{600}$ of 0.7 , the temperature was reduced to $16^{\circ} \mathrm{C}$ and expression was induced by addition of $1 \mathrm{mM}$ IPTG for $18 \mathrm{~h}$. Cells were pelleted at $3000 \times \mathrm{g}$ for $15 \mathrm{~min}$ at RT, resuspended in GST-lysis buffer (50 mM Tris- $\mathrm{HCl} \mathrm{pH} \mathrm{7.5,150} \mathrm{mM} \mathrm{NaCl,} \mathrm{5 \%} \mathrm{glycerol,} 1 \%$ Triton X-100, $1 \mathrm{mM}$ PMSF, $1 \mathrm{mM}$ DTT, and complete ${ }^{\mathrm{TM}}$ protease inhibitor cocktail [Roche]), and lysed by sonication on ice. Cell lysates were cleared by centrifugation at $16,000 \times \mathrm{g}$ for $10 \mathrm{~min}$ at $4{ }^{\circ} \mathrm{C}$. The supernatant was incubated with Glutathione (GSH) Sepharose $4 \mathrm{~B}$ beads (GE Healthcare) for $1 \mathrm{~h}$ rotating at $4^{\circ} \mathrm{C}$. The beads were washed three times by pelleting at $300 \times \mathrm{g}$ for $30 \mathrm{sec}$ at $4^{\circ} \mathrm{C}$ and resuspension in GST-wash buffer I (50 mM Tris- $\mathrm{HCl}$ pH 7.5, $150 \mathrm{mM} \mathrm{NaCl}, 5 \%$ glycerol, $1 \%$ Triton X-100, $1 \mathrm{mM}$ DTT and complete ${ }^{\mathrm{TM}}$ protease inhibitor cocktail [Roche]), and once with 1x PBS $\mathrm{pH}$ 7.4. The beads were incubated with cleared Sf9 insect cell lysate from 8xHISAtg11 expressing cells or noninfected control cells, and incubated for $1 \mathrm{~h}$ rotating at $4{ }^{\circ} \mathrm{C}$. The beads were washed three times with GST-wash buffer II ( $50 \mathrm{mM}$ Tris$\mathrm{HCl} \mathrm{pH} \mathrm{7.5,} 150 \mathrm{mM} \mathrm{NaCl}, 5 \%$ glycerol, $0.1 \%$ Triton X-100, 0.5\% Tween-20, and $1 \mathrm{mM}$ DTT and complete ${ }^{\mathrm{TM}}$ protease inhibitor cocktail [Roche]), and once with GST-wash buffer III (1x PBS, pH 7.4, 10\% glycerol, 0.5\% Tween-20, 1 mM DTT). The beads were resuspended in urea-loading buffer, boiled and analyzed by SDSPAGE and western blotting.

Live-cell imaging. Exponentially growing cells were placed on $35 \mathrm{~mm}$ glass bottom dishes (D35-20 1.5-N, In Vitro Scientific) pretreated with concanavalin A type IV $(1 \mathrm{mg} / \mathrm{ml}$, Sigma-Aldrich), and live-cell imaging was performed at RT.

Fluorescent microscopy images were recorded with a DeltaVision Ultra High Resolution microscope (GE Healthcare, Applied Precision) equipped with an UPlanSApo 100x/1.4 oil objective (Olympus), an sCMOS pco.edge camera (PCO), and a seven channel solid state light source (Lumencor) (Figs. 1a, b, d-g, 2a, b, and Supplementary Figs. 1a-d, 2a, b, 3b, 4d, e, 5c, d, 6d, 8b, e, f, 9b, c); or with a PersonalDeltaVision microscope (GE Healthcare, Applied Precision) equipped with an UPlanSApo 100× oil/1.4 oil objective (Olympus), a CoolSNAP HQ2 Monochrome CCD camera (Photometrics), and a seven-color InsightSSI solid state illumination unit (GE Healthcare, Applied Precision) (Fig. 2f and Supplementary Fig. 3a); or with an AxioObserver $\mathrm{Zl}$ inverted microscope (ZEISS) equipped with an EC Plan-Neofluar 100x/1.3 oil M27 objective (ZEISS), a CoolSnap HQ2 Monochrome CCD camera (Photometrics), and a SOLA 6-LCR-SB light source (Lumencor) with the VisiView software (Visitron Systems) (Figs. 3c-g, 4b-e, g, 5a-d, 6a-e, 7a, b, and Supplementary Figs. 4b, c, 5a, b, e-g, 6a-c, e, 7a, b, 8a, c, d, 9a).
Raw microscopy images acquired with the PersonalDeltaVision microscope or the DeltaVision Ultra High Resolution microscope were deconvolved using the softWorX deconvolution plugin (version R6.1.1 and version 7.2.1, respectively). Image analysis was performed using $\mathrm{FIJI}^{61}$. Images from each figure panel were taken with the same imaging setup and are shown with the same contrast settings, unless stated otherwise. Single focal planes of representative images are shown. For quantification three independent replicates were analyzed and manual counting was performed blindly after randomizing image names.

Subcellular positioning of mTagBFP-Apel or GFP- $\mu$ NS particles was investigated by analyzing their localization in regard to the vacuole, by staining vacuoles with the $\mathrm{FM}^{\mathrm{TM}}$ 4-64 (Thermo Fisher Scientific) dye or by using the genomically tagged vacuole marker protein Vac8-mCherry, Vph1-4xmCherry or Sna3-4xmCherry as indicated. Images were generated by collecting a $z$-stack of 21 pictures with focal planes $0.25 \mu \mathrm{m}$ apart or 30 pictures with focal planes $0.20 \mu \mathrm{m}$ apart. (Figs. 1a, b, d-g, 2a, b, f, and Supplementary Figs. 1a, b, 2a, b, 3a, b).

Size quantification of $\mu$ NS particles was performed in FIJI on a maximumintensity z-projection. To generate binarized masks, the same intensity threshold was applied to all images, which were then subjected to particle analysis, using a minimal size of 8 pixels and a circularity between 0.5 and 1 . To extract particle sizes, the masks were applied on the z-projected images (Fig. 1d, Supplementary Fig. 1b).

Recruitment of GFP tagged prey proteins to oligomer-tethered bait proteins was analyzed by quantifying the number of cells with GFP puncta, and association of oligomer-tethered ot-Vac $8 \Delta \mathrm{N}-\mathrm{GFP}$ with Atg11-BFP- $\mu$ NS particles was analyzed by counting the number of BFP-puncta overlapping with GFP puncta. Images were generated by collecting a $\mathrm{z}$-stack of 11 pictures with focal planes $0.25 \mu \mathrm{m}$ apart

(Figs. 3c-g, 5a, b, and Supplementary Figs. 4b-e, 6a-c, e).

Quantitative analysis of PASs, forming autophagosomes or completed autophagosomes was performed by counting the number of cells with GFP-Atg8 or Atg14-3xGFP puncta, or by counting the number of mTagBFP2-Apel puncta overlapping with Atg9-GFP puncta. Images were generated by collecting a $\mathrm{z}$-stack of 21 pictures with focal planes $0.25 \mu \mathrm{m}$ apart (Fig. $4 \mathrm{~b}-\mathrm{d}$, and Supplementary Fig. 5a-e).

Vacuole association of Atg14-3xGFP or Vps15-GFP was investigated by staining vacuoles with the FM ${ }^{\mathrm{TM}} 4-64$ (Thermo Fisher Scientific) dye or by using the genomically tagged vacuole marker protein Vph1-4xmCherry. Images were generated on one focal plane (Fig. 4e, g, and Supplementary Figs. 5f, g, 6d).

Vacuole association of artificially vacuole tethered mScarlet-Atg11 or Atg14 GFP was investigated by using the genomically tagged vacuole marker protein Vph1-GFP or Vph1-4xmCherry, respectively. Images were generated by collecting a z-stack of 21 pictures with focal planes $0.25 \mu \mathrm{m}$ apart (Supplementary Fig. 7a, b).

Association of Atg14-GFP with mScarlet-Atg11 clustered on mTagBFP2-Ape oligomers was analyzed by counting the number of mScarlet-mTagBFP2 doublepositive puncta overlapping with GFP puncta. Images were generated by collecting a z-stack of 11 pictures with focal planes $0.25 \mu \mathrm{m}$ apart (Fig. 5c, d).

Nucleus association of artificially nucleus-tethered nt-mScarlet-Vac $8 \Delta \mathrm{N}$ was investigated by staining of the nucleus with the Hoechst 33258 (Sigma) dye. Images were generated by collecting a z-stack of 11 pictures with focal planes $0.25 \mu \mathrm{m}$ apart (Fig. 6a).

Subcellular positioning of GFP-Atg11 puncta or Vps15-GFP signal was investigated by analyzing their localization in regard to the nuclear membrane, marked by expression of nucleus tethered nt-mScarlet-Vac $8 \Delta \mathrm{N}$ or nt-mScarlet. Cells not containing nuclear mScarlet signal were excluded from the analysis. Images were generated by collecting a $\mathrm{z}$-stack of 11 pictures with focal planes $0.25 \mu \mathrm{m}$ apart (Fig. 6b, c, and Supplementary Fig. S8a, c). 
Quantitative analysis of ectopic PASs, forming autophagosomes or completed autophagosomes was performed by counting the number of cells with GFP-Atg8, Atg2-GFP puncta, or by counting the number of mTagBFP2-Ape1 puncta overlapping with Atg2-GFP puncta. Cells not containing nuclear mScarlet signal were excluded from the analysis. Images were generated by collecting a $z$-stack of 11 pictures with focal planes $0.25 \mu \mathrm{m}$ apart (Fig. 6d, e, Supplementary and Figs. 8d-f, 9a-c).

Transport of mTagBFP2-Ape1 into the vacuole was analyzed by using the genomically tagged vacuole marker protein Vph1-GFP and counting the number of cells containing BFP signal within the vacuolar lumen. Cells not containing nuclear $\mathrm{mScarlet}$ signal were excluded from the analysis. Images were generated on one focal plane, except for the BFP channel, 7 pictures with focal planes $0.25 \mu \mathrm{m}$ apart were collected and the intensity averaged. (Fig. 7a, b).

Electron microscopy. Fifteen $\mathrm{OD}_{600}$ units of cells were harvested by centrifugation $(1800 \times \mathrm{g}, 5 \mathrm{~min}, \mathrm{RT})$. Cells were washed in distilled $\mathrm{H}_{2} \mathrm{O}$ and pelleted by centrifugation $(1800 \times \mathrm{g}, 5 \mathrm{~min}, \mathrm{RT})$. Cells were resuspended in $3 \mathrm{ml}$ of freshly prepared ice-cold $1.5 \% \mathrm{KMnO}_{4}$ (Sigma) and transferred into two $1.5 \mathrm{ml}$ microfuge tubes. After topping up the tube with the same solution to exclude air, samples were mixed for $30 \mathrm{~min}$ rotating at $4^{\circ} \mathrm{C}$. After centrifugation $(1400 \times \mathrm{g}, 3 \mathrm{~min}$, $4{ }^{\circ} \mathrm{C}$ ), the $1.5 \% \mathrm{KMnO}_{4}$ incubation was repeated once more before washing the pellets five times with $1 \mathrm{ml}$ of $\mathrm{H}_{2} \mathrm{O}$. Permanganate-fixed cells were dehydrated stepwise with increasing concentrations of acetone $(10 \%, 30 \%, 50 \%, 70 \%, 90 \%$, $95 \%$ and three times 100\%). Each incubation step was performed for 20 min rotating at RT, in-between each step cells were pelleted by centrifugation $(1400 \times \mathrm{g}, 3 \mathrm{~min}, \mathrm{RT})$. Cell pellets were resuspended in 33\% Spurr's resin in acetone and mixed for $1 \mathrm{~h}$ rotating at RT. Cells were pelleted $(7600 \times \mathrm{g}, 3 \mathrm{~min}, \mathrm{RT})$ and incubated in $100 \%$ freshly made Spurr's resin overnight rotating at RT. This operation was repeated the following day, over the day, after centrifugation of the overnight incubation $(9000 \times \mathrm{g}, 5 \mathrm{~min}, \mathrm{RT})$. The Spurr's resin mixture was prepared by mixing $10 \mathrm{~g}$ of 4 -vinylcyclohexene dioxide (or ERL4206), $4 \mathrm{~g}$ of epichlorohydrin-polyglycol epoxy (DER) resin 736, $26 \mathrm{~g}$ of (2-nonen-1-yl)succinic anhydride (NSA), and $0.4 \mathrm{~g}$ of N,N-diethylethanolamine (all from Sigma). Afterward, the cell and Spurr's mixture was transferred to size 00 embedding capsules (Electron Microscopy Science), and cells were pelleted by centrifugation $(9000 \times \mathrm{g}, 5 \mathrm{~min}, \mathrm{RT})$. Embedding capsules were topped up with $100 \%$ Spurr's and baked for a minimum of 3 days at $60^{\circ} \mathrm{C}$.

Thin sections of $\sim 55 \mathrm{~nm}$ in thickness were cut using an ultramicrotome (Leica Microsystems). Sections were collected on formvar carbon-coated 50 mesh copper grids (EMS) and stained with a filtered lead-citrate solution ( $80 \mathrm{mM}$ lead nitrate, $120 \mathrm{mM}$ sodium citrate $\mathrm{pH}$ 12) for $2 \mathrm{~min}$ at RT. Sections were viewed in a CM100bio TEM (FEI, Eindhoven). Contact between GFP- $\mu$ NS or Atg11-GFP- $\mu$ NS particles with the vacuolar membrane was analyzed by manual quantification of randomly selected cell profiles on three independent grids per conditions.

Statistics and reproducibility. To assess statistical significance, two-tailed unpaired $t$-tests were performed. The underlying data of box plots and bar charts presented in this study and the exact $p$ values, means, and standard deviations are provided in the source data.

Reporting summary. Further information on research design is available in the Nature Research Reporting Summary linked to this article.

\section{Data availability}

All relevant data supporting the key findings of this study are available within the article and its Supplementary Information files or from the corresponding author upon reasonable request. Raw data underlying the bar graphs and uncropped western blots are provided in the Source Data file. Source data are provided with this paper.

Received: 19 May 2021; Accepted: 17 November 2021;

Published online: 10 December 2021

\section{References}

1. Hollenstein, D. M. \& Kraft, C. Autophagosomes are formed at a distinct cellular structure. Curr. Opin. Cell Biol. 65, 50-57 (2020).

2. Nakatogawa, H. Mechanisms governing autophagosome biogenesis. Nat. Rev. Mol. Cell Biol. 21, 439-458 (2020).

3. Kirkin, V. \& Rogov, V. V. A diversity of selective autophagy receptors determines the specificity of the autophagy pathway. Mol. Cell 76, 268-285 (2019).

4. Zellner, S., Schifferer, M. \& Behrends, C. Systematically defining selective autophagy receptor-specific cargo using autophagosome content profiling. Mol. Cell 81, 1337-1354.e8 (2021).
5. Shintani, T., Huang, W.-P., Stromhaug, P. E. \& Klionsky, D. J. Mechanism of cargo selection in the cytoplasm to vacuole targeting pathway. Developmental Cell 3, 825-837 (2002).

6. Stjepanovic, G., Baskaran, S., Lin, M. G. \& Hurley, J. H. Vps34 kinase domain dynamics regulate the autophagic PI 3-kinase complex. Mol. Cell 67, 528-534.e3 (2017).

7. Kamber, R. A., Shoemaker, C. J. \& Denic, V. Receptor-bound targets of selective autophagy use a scaffold protein to activate the Atg1 kinase. Mol. Cell 59, 372-381 (2015)

8. Torggler, R. et al. Two independent pathways within selective autophagy converge to activate Atg1 kinase at the vacuole. Mol. Cell 64, 221-235 (2016).

9. Yorimitsu, T. \& Klionsky, D. J. Atg11 links cargo to the vesicle-forming machinery in the cytoplasm to vacuole targeting pathway. $M B O C \mathrm{16}$, 1593-1605 (2005).

10. Hara, T. et al. FIP200, a ULK-interacting protein, is required for autophagosome formation in mammalian cells. J. Cell Biol. 181, 497-510 (2008).

11. Nishimura, T. et al. Autophagosome formation is initiated at phosphatidylinositol synthase-enriched ER subdomains. EMBO J. 36, 1719-1735 (2017)

12. Turco, E. et al. FIP200 claw domain binding to $\mathrm{p} 62$ promotes autophagosome formation at ubiquitin condensates. Mol. Cell 74, 330-346.e11 (2019).

13. Cheong, H. et al. Atg17 regulates the magnitude of the autophagic response. $M B o C$ 16, 3438-3453 (2005).

14. Pan, X. et al. Nucleus-vacuole junctions in saccharomyces cerevisiae are formed through the direct interaction of Vac8p with Nvj1p. MBoC 11, 2445-2457 (2000).

15. Roberts, P. et al. Piecemeal microautophagy of nucleus in Saccharomyces cerevisiae. MBoC 14, 129-141 (2002).

16. Scott, S. V. et al. Apg13p and Vac8p are part of a complex of phosphoproteins that are required for cytoplasm to vacuole targeting. J. Biol. Chem. 275, 25840-25849 (2000).

17. Veit, M., Laage, R., Dietrich, L., Wang, L. \& Ungermann, C. Vac8p release from the SNARE complex and its palmitoylation are coupled and essential for vacuole fusion. ЕMBO J. 20, 3145-3155 (2001).

18. Wang, Y.-X., Catlett, N. L. \& Weisman, L. S. Vac8p, a vacuolar protein with armadillo repeats, functions in both vacuole inheritance and protein targeting from the cytoplasm to vacuole. J. Cell Biol. 140, 1063-1074 (1998).

19. Fujioka, Y. et al. Phase separation organizes the site of autophagosome formation. Nature 578, 301-305 (2020).

20. Gatica, D., Wen, X., Cheong, H. \& Klionsky, D. J. Vac8 determines phagophore assembly site vacuolar localization during nitrogen starvationinduced autophagy. Autophagy 17, 1636-48 (2021).

21. Hollenstein, D. M. et al. Vac8 spatially confines autophagosome formation at the vacuole in S. cerevisiae. J. Cell Sci. 132 jcs235002 (2019).

22. Munzel, L. et al. Atg21 organizes Atg8 lipidation at the contact of the vacuole with the phagophore. Autophagy 17, 1458-78 (2020).

23. Alam, J. M. \& Noda, N. N. In vitro reconstitution of autophagic processes. Biochem Soc. Trans. 48, 2003-2014 (2020).

24. Moparthi, S. B. \& Wollert, T. Reconstruction of destruction - in vitro reconstitution methods in autophagy research. J. Cell Sci. 132 jcs223792 (2019).

25. Suzuki, K., Kamada, Y. \& Ohsumi, Y. Studies of cargo delivery to the vacuole mediated by autophagosomes in Saccharomyces cerevisiae. Developmental Cell 3, 815-824 (2002)

26. Munder, M. C. et al. A pH-driven transition of the cytoplasm from a fluid- to a solid-like state promotes entry into dormancy. eLife 5, e09347 (2016).

27. Pan, X. \& Goldfarb, D. S. YEB3/VAC8 encodes a myristylated armadillo protein of the Saccharomyces cerevisiae vacuolar membrane that functions in vacuole fusion and inheritance. J. Cell Sci. 111, 2137-2147 (1998).

28. Pfaffenwimmer, T. et al. Hrr25 kinase promotes selective autophagy by phosphorylating the cargo receptor Atg19. EMBO Rep. 15, 862-870 (2014).

29. Watanabe, Y. et al. Selective transport of alpha-mannosidase by autophagic pathways: structural basis for cargo recognition by Atg19 and Atg34. J. Biol. Chem. 285, 30026-30033 (2010).

30. Erlendsson, S. \& Teilum, K. Binding revisited-avidity in cellular function and signaling. Front. Mol. Biosci., 10.3389/fmolb.2020.615565 (2021).

31. Kihara, A., Noda, T., Ishihara, N. \& Ohsumi, Y. Two distinct Vps34 phosphatidylinositol 3-kinase complexes function in autophagy and carboxypeptidase Y sorting in Saccharomyces cerevisiae. J. Cell Biol. 152, 519-530 (2001)

32. Cao, Y. \& Klionsky, D. J. New insights into autophagy using a multiple knockout strain. Autophagy 4, 1073-1075 (2008).

33. Jeong, H. et al. Mechanistic insight into the nucleus-vacuole junction based on the Vac8p-Nvjlp crystal structure. PNAS 114, E4539-E4548 (2017).

34. Kvam, E. \& Goldfarb, D. S. Structure and function of nucleus-vacuole junctions: outer-nuclear-membrane targeting of Nvjlp and a role in tryptophan uptake. J. Cell Sci. 119, 3622-3633 (2006). 
35. Kobayashi, T., Suzuki, K. \& Ohsumi, Y. Autophagosome formation can be achieved in the absence of Atg18 by expressing engineered PAS-targeted Atg2. FEBS Lett. 586, 2473-2478 (2012).

36. Obara, K., Sekito, T., Niimi, K. \& Ohsumi, Y. The Atg18-Atg2 complex is recruited to autophagic membranes via phosphatidylinositol 3-phosphate and exerts an essential function. J. Biol. Chem. 283, 23972-23980 (2008).

37. Kirisako, T. et al. Formation process of autophagosome is traced with Apg8/ Aut7p in yeast. J. Cell Biol. 147, 435-446 (1999).

38. Yamamoto, H. et al. The intrinsically disordered protein Atg13 mediates supramolecular assembly of autophagy initiation complexes. Dev. Cell $\mathbf{3 8}$ 86-99 (2016)

39. Agudo-Canalejo, J. et al. Wetting regulates autophagy of phase-separated compartments and the cytosol. Nature 591, 142-146 (2021).

40. Wilfling, F. et al. A selective autophagy pathway for phase-separated endocytic protein deposits. Mol. Cell 80, 764-778.e7 (2020).

41. Yamasaki, A. et al. Liquidity is a critical determinant for selective autophagy of protein condensates. Mol. Cell, https://doi.org/10.1016/j.molcel.2019.12.026 (2020).

42. Itakura, E., Kishi-Itakura, C., Koyama-Honda, I. \& Mizushima, N. Structures containing Atg9A and the ULK1 complex independently target depolarized mitochondria at initial stages of Parkin-mediated mitophagy. J. Cell Sci. 125, 1488-1499 (2012).

43. Matsunaga, K. et al. Autophagy requires endoplasmic reticulum targeting of the PI3-kinase complex via Atg14L. J. Cell Biol. 190, 511-521 (2010).

44. Matsunaga, K. et al. Two Beclin 1-binding proteins, Atg14L and Rubicon, reciprocally regulate autophagy at different stages. Nat. Cell Biol. 11, 385-396 (2009).

45. Mercer, T. J., Gubas, A. \& Tooze, S. A. A molecular perspective of mammalian autophagosome biogenesis. J. Biol. Chem. 293, 5386-5395 (2018).

46. Lei, Y. et al. Autophagic elimination of ribosomes during spermiogenesis provides energy for flagellar motility. Dev. Cell 56, 2313-2328.e7 (2021).

47. Dawaliby, R. \& Mayer, A. Microautophagy of the nucleus coincides with a vacuolar diffusion barrier at nuclear-vacuolar junctions. $M B o C$ 21, 4173-4183 (2010).

48. Kornmann, B. et al. An ER-mitochondria tethering complex revealed by a synthetic biology screen. Science 325, 477-481 (2009).

49. Toulmay, A. \& Prinz, W. A. Direct imaging reveals stable, micrometer-scale lipid domains that segregate proteins in live cells. J. Cell Biol. 202, 35-44 (2013).

50. Suzuki, K., Akioka, M., Kondo-Kakuta, C., Yamamoto, H. \& Ohsumi, Y. Fine mapping of autophagy-related proteins during autophagosome formation in Saccharomyces cerevisiae. J. Cell Sci. 126, 2534-2544 (2013).

51. Ravenhill, B. J. et al. The cargo receptor NDP52 initiates selective autophagy by recruiting the ULK complex to cytosol-invading bacteria. Mol. Cell 74, 320-329.e6 (2019).

52. Vargas, J. N. S. et al. Spatiotemporal control of ULK1 activation by NDP52 and TBK1 during selective autophagy. Mol. Cell 74, 347-362.e6 (2019).

53. Lu, K., den Brave, F. \& Jentsch, S. Receptor oligomerization guides pathway choice between proteasomal and autophagic degradation. Nat. Cell Biol. 19, 732-739 (2017).

54. Lu, K., Psakhye, I. \& Jentsch, S. Autophagic clearance of PolyQ proteins mediated by ubiquitin-Atg8 adaptors of the conserved CUET protein family. Cell 158, 549-563 (2014).

55. Pohl, C. \& Dikic, I. Cellular quality control by the ubiquitin-proteasome system and autophagy. Science 366, 818-822 (2019).

56. Sawa-Makarska, J. et al. Cargo binding to Atg19 unmasks additional Atg8 binding sites to mediate membrane-cargo apposition during selective autophagy. Nat. Cell Biol. 16, 425-433 (2014).

57. Janke, C. et al. A versatile toolbox for PCR-based tagging of yeast genes: new fluorescent proteins, more markers and promoter substitution cassettes. Yeast 21, 947-962 (2004).

58. Longtine, M. S. et al. Additional modules for versatile and economical PCRbased gene deletion and modification in Saccharomyces cerevisiae. Yeast 14, 953-961 (1998).

59. Khmelinskii, A., Meurer, M., Duishoev, N., Delhomme, N. \& Knop, M. Seamless gene tagging by endonuclease-driven homologous recombination. PLoS ONE 6, e23794 (2011).

60. Weissmann, F. et al. biGBac enables rapid gene assembly for the expression of large multisubunit protein complexes. PNAS 113, E2564-E2569 (2016).

61. Schindelin, J. et al. Fiji: an open-source platform for biological-image analysis. Nat. Methods 9, 676-682 (2012).

\section{Acknowledgements}

We would like to thank Dominik Kaiser for help with insect cell expression, Irmgard Fischer and the Max Perutz Labs BioOptics facility for help with microscopy, Thomas Leonard and Linda Trübestein for help with insect cell culture, Serge Pelet for plasmids, Chris Meisinger for antibodies, Daniel Klionsky for the multiple-knock out strain and antibodies, and Life Science Editors (Angela Andersen) for help with paper editing. Electron microscopy imaging was performed at the UMCG microscopy and imaging center (UMIC). The Kraft laboratory has received funding from the Deutsche Forschungsgemeinschaft (DFG, German Research Foundation), Project ID 409673687 (to C.K.); SFB 1381 (Project-ID 403222702; to C.K.); SFB 1177 (Project-ID 259130777; to C.K., N.C.); under Germany's Excellence Strategy (CIBSS-EXC-2189- Project ID 390939984; to C.K.); from the European Research Council (ERC) under the European Union's Horizon 2020 research and innovation programme (grant agreement No 769065, to C.K., D.M.H., and M.L.); from the FWF Austrian Science Fund (grant number P25522-B20; to C.K., D.S., and W1261, to C.K., D.M.H.). This work was further supported by ENW KLEIN-1 (OCENW.KLEIN.118; to F.R.) and ZonMW TOP (91217002, to F.R.) grants, by an ALW Open Programme (ALWOP.355; to M.M.), and by a Marie Skłodowska-Curie ETN grant under the European Union's Horizon 2020 Research and Innovation Programme (Grant Agreement No 765912, to C.K., M.L., and F.R.). This work reflects only the authors' view and the European Union's Horizon 2020 research and innovation programme is not responsible for any use that may be made of the information it contains.

\section{Author contributions}

Conceptualization D.M.H., M.L., and C.K.; Methodology D.M.H., M.L., M.M., F.R., H.M and C.K.; Validation D.M.H., M.L., N.K., D.S., M.M., F.R., H.M., and C.K.; Investigation D.M.H., M.L., N.K., D.S., H.M. and M.M.; Writing-Original Draft C.K.; WritingReview and Editing D.M.H., M.L., F.R. and C.K.; Visualization D.M.H., M.L., M.M. and C.K.; Supervision F.R. and C.K.; Project Administration D.M.H., M.L., and C.K.; Funding Acquisition F.R. and C.K.

\section{Funding}

Open Access funding enabled and organized by Projekt DEAL.

\section{Competing interests}

The authors declare no competing interests.

\section{Additional information}

Supplementary information The online version contains supplementary material available at https://doi.org/10.1038/s41467-021-27420-3.

Correspondence and requests for materials should be addressed to Claudine Kraft.

Peer-review information Nature Communications thanks Kuninori Suzuki, Thomas Wollert and the other anonymous reviewer(s) for their contribution to the peer review of this work. Peer-reviewer reports are available.

Reprints and permission information is available at http://www.nature.com/reprints

Publisher's note Springer Nature remains neutral with regard to jurisdictional claims in published maps and institutional affiliations.

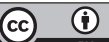

Open Access This article is licensed under a Creative Commons Attribution 4.0 International License, which permits use, sharing, adaptation, distribution and reproduction in any medium or format, as long as you give appropriate credit to the original author(s) and the source, provide a link to the Creative Commons license, and indicate if changes were made. The images or other third party material in this article are included in the article's Creative Commons license, unless indicated otherwise in a credit line to the material. If material is not included in the article's Creative Commons license and your intended use is not permitted by statutory regulation or exceeds the permitted use, you will need to obtain permission directly from the copyright holder. To view a copy of this license, visit http://creativecommons.org/ licenses/by/4.0/.

(c) The Author(s) 2021 